\title{
Low-energy Coulomb excitation of neutron-rich zinc isotopes
}

J. Van de Walle, ${ }^{1,2}$ F. Aksouh,,${ }^{1,3}$ T. Behrens, ${ }^{4}$ V. Bildstein, ${ }^{4,5}$ A. Blazhev, ${ }^{6}$ J. Cederkäll, ${ }^{2}$ E. Clément,,${ }^{2,3}$ T. E. Cocolios, ${ }^{1}$ T. Davinson, ${ }^{7}$ P. Delahaye, ${ }^{2}$ J. Eberth, ${ }^{6}$ A. Ekström, ${ }^{8}$ D. V. Fedorov, ${ }^{9}$ V. N. Fedosseev, ${ }^{2}$ L. M. Fraile,,${ }^{2,10}$ S. Franchoo, ${ }^{2}$ R. Gernhauser ${ }^{4}$ G. Georgiev, ${ }^{2,11}$ D. Habs,${ }^{12}$ K. Heyde,${ }^{13}$ G. Huber,${ }^{14}$ M. Huyse,${ }^{1}$ F. Ibrahim, ${ }^{15}$ O. Ivanov, ${ }^{1}$ J. Iwanicki, ${ }^{16}$ J. Jolie, ${ }^{6}$ O. Kester, ${ }^{17}$ U. Köster, ${ }^{2,18}$ T. Kröll, ${ }^{4}$ R. Krücken, ${ }^{4}$ M. Lauer,${ }^{5}$ A. F. Lisetskiy, ${ }^{17}$ R. Lutter, ${ }^{12}$ B. A. Marsh, ${ }^{2}$ P. Mayet, ${ }^{1}$ O. Niedermaier, ${ }^{5}$ M. Pantea, ${ }^{19}$ R. Raabe, ${ }^{1}$ P. Reiter, ${ }^{6}$ M. Sawicka, ${ }^{1}$ H. Scheit,${ }^{5}$ G. Schrieder, ${ }^{19}$ D. Schwalm, ${ }^{5}$ M. D. Seliverstov, ${ }^{9,14}$ T. Sieber, ${ }^{2}$ G. Sletten, ${ }^{20}$ N. Smirnova, ${ }^{13,21}$ M. Stanoiu, ${ }^{17,22}$ I. Stefanescu, ${ }^{1}$ J.-C. Thomas,,${ }^{1,23}$ J. J. Valiente-Dobón, ${ }^{24}$ P. Van Duppen, ${ }^{1}$ D. Verney, ${ }^{15}$ D. Voulot, ${ }^{2}$ N. Warr, ${ }^{6}$ D. Weisshaar, ${ }^{6}$ F. Wenander, ${ }^{2}$

B. H. Wolf, ${ }^{2}$ and M. Zielińska ${ }^{3,16}$

${ }^{1}$ Instituut voor Kern-en Stralingsfysica, K. U. Leuven, Leuven, Belgium

${ }^{2}$ ISOLDE, CERN, Geneva, Switzerland

${ }^{3}$ CEA Saclay, DAPNIA/SPhN, Gif-sur-Yvette, France

${ }^{4}$ Physik Department E12, Technische Universität München, Garching, Germany

${ }^{5}$ Max-Planck-Institut für Kernphysik, Heidelberg, Germany

${ }^{6}$ Institut für Kernphysik, Universität Köln, Köln, Germany

${ }^{7}$ University of Edinburgh, Edinburgh, United Kingdom

${ }^{8}$ Physics Department, University of Lund, Lund, Sweden

${ }^{9}$ Department of High Energy Physics, Petersburg Nuclear Physics Institute, Gatchina, Russia

${ }^{10}$ Universidad Complutense, Madrid, Spain

${ }^{11}$ CSNSM, IN2P3-CNRS, Université Paris-Sud, Orsay, France

${ }^{12}$ Ludwig-Maximilians-Universität, München, Germany

${ }^{13}$ Vakgroep Subatomaire en Stralingsfysica, Universiteit Gent, Gent, Belgium

${ }^{14}$ Institut für Physik, Johannes Gutenberg Universität Mainz, Mainz, Germany

${ }^{15}$ Institut de Physique Nucléaire, IN2P3-CNRS, Orsay, France

${ }^{16}$ Heavy Ion Laboratory, University of Warsaw, Warsaw, Poland

${ }^{17}$ Gesellschaft für Schwerionenforschung mbH, Darmstadt, Germany

${ }^{18}$ Institut Laue-Langevin, Grenoble, France

${ }^{19}$ Institut für Kernphysik, Technische Universität Darmstadt, Darmstadt, Germany

${ }^{20}$ Physics Department, University of Copenhagen, Denmark

${ }^{21}$ Centre d'Etudes Nucléaires de Bordeaux-Gradignan, Université Bordeaux, Gradignan Cedex, France

${ }^{22}$ Institutul National de Fizica si Inginerie Nucleara, IFIN-HH, Bucuresti-Magurele, Romania

${ }^{23}$ GANIL, IN2P3-CNRS-CEA, Caen, France

${ }^{24}$ Instituto Nazionale di Fisica Nucleare, Laboratori Nazionali di Legnaro, Legnaro, Italy

(Received 25 August 2008; published 22 January 2009)

\begin{abstract}
At the radioactive ion beam facility REX-ISOLDE, neutron-rich zinc isotopes were investigated using lowenergy Coulomb excitation. These experiments have resulted in $B\left(E 2,2_{1}^{+} \rightarrow 0_{1}^{+}\right)$values in ${ }^{74-80} \mathrm{Zn}, B\left(E 2,4_{1}^{+} \rightarrow\right.$ $2_{1}^{+}$) values in ${ }^{74,76} \mathrm{Zn}$ and the determination of the energy of the first excited $2_{1}^{+}$states in ${ }^{78,80} \mathrm{Zn}$. The zinc isotopes were produced by high-energy proton- $(A=74,76,80)$ and neutron- $(A=78)$ induced fission of ${ }^{238} \mathrm{U}$, combined with selective laser ionization and mass separation. The isobaric beam was postaccelerated by the REX linear accelerator and Coulomb excitation was induced on a thin secondary target, which was surrounded by the MINIBALL germanium detector array. In this work, it is shown how the selective laser ionization can be used to deal with the considerable isobaric beam contamination and how a reliable normalization of the experiment can be achieved. The results for zinc isotopes and the $N=50$ isotones are compared to collective model predictions and state-of-the-art large-scale shell-model calculations, including a recent empirical residual interaction constructed to describe the present experimental data up to 2004 in this region of the nuclear chart.
\end{abstract}

DOI: 10.1103/PhysRevC.79.014309

PACS number(s): 23.20.-g, 21.60.Cs, 25.70.De, 27.50.+e

\section{INTRODUCTION}

The nuclei near $Z=28$ form a region of intensive research in both their experimental and theoretical properties. The nickel isotopes cover nuclei encompassing three doubly closed shell numbers, i.e., $N=28, N=40$, and $N=50$. There is a particular interest in possible variations in both the position (neutron number $N$ and proton number $Z$ ) and size (energy gap) of the traditional shell gaps because the ratio $N / Z$ for nickel isotopes ranges from 1 near stability to 1.8 in the most neutron-rich nickel nuclei. Recent review articles [1,2] study proton and/or neutron single-particle energy variations (the so-called monopole energy shift) for the whole nuclear mass region, with particular attention to the region near $Z=28$. There are by now convincing experimental results for a changing shell structure in lighter nuclei (e.g., $N=8$ [3], $N=20$ [4], $N=28$ [5]); however, in heavy $r$-process waiting 
point nuclei with $N=82$, contradictory results have been reported [6,7].

Even though the origin of the changing shell structure is determined by the average nucleon-nucleon matrix elements [8-10] that describe the relative self-energy Hartree-Fock(Bogoliubov) $[\mathrm{HF}(\mathrm{B})]$ variations, it has been shown that the proton-neutron tensor nucleon-nucleon force component is one of the dominant components [11,12] responsible for the observed single-particle variations. This tensor force implies in particular an attractive contribution to the energy of a proton moving in the $\pi 1 f_{5 / 2}(l=j-1 / 2)$ orbital when filling the $v 1 g_{9 / 2}(l=j+1 / 2)$ orbital while a repulsive contribution to the energy of a proton moving in the $\pi 1 f_{7 / 2}(l=j+1 / 2)$ spin-orbit partner orbital results, when filling the $v 1 g_{9 / 2}$ orbital. Experimental hints for a corresponding decrease in the $\Delta\left(\epsilon_{\pi 1 f_{5 / 2}}-\epsilon_{\pi 1 f_{7 / 2}}\right)$ proton energy gap is found from the increased collectivity in ${ }^{70} \mathrm{Ni}$ [13].

Going beyond the effect of single-particle energy variations as given through the monopole term, more detailed insight and results can be obtained only when large-scale shell model calculations are carried out. These calculations should at least be performed in the valence space consisting of $2 p_{3 / 2} 1 f_{5 / 2} 2 p_{1 / 2}-1 g_{9 / 2}$ orbitals for both protons and neutrons. Moreover, constructing a nucleon-nucleon interaction for this model space, which should be able to describe nuclei and their properties situated between ${ }^{56} \mathrm{Ni}$ and ${ }^{100} \mathrm{Sn}$, is one of the current paradigms in nuclear structure. The above space should even be extended to include the proton $1 f_{7 / 2}$ orbital to allow for proton particle-hole excitations across $Z=28$ if one tries to understand the polarizability of the $Z=28$ shell when neutrons start filling orbitals between $N=28$ and $N=50$. It should have become clear that to reach a stage of predicting nuclear properties correctly, experimental information on the most exotic nuclei $(Z \approx 28, N \approx 50$ ) is highly important to constrain future effective interactions.

In the present study, we report on the Coulomb excitation experiments performed at REX-ISOLDE with postaccelerated beams of neutron-rich even-even zinc isotopes ${ }^{74,76,78,80} \mathrm{Zn}$. With two protons outside the $Z=28$ proton shell and the gradual occupation of neutrons in the $1 g_{9 / 2}$ orbital, these zinc isotopes are ideally suited to study the evolution of the $Z=28$ shell gap and the stability of the $N=50$ neutron shell gap near ${ }^{78} \mathrm{Ni}$. The experiments have resulted in $B\left(E 2,2_{1}^{+} \rightarrow\right.$ $0_{1}^{+}$) values in ${ }^{74-80} \mathrm{Zn}$ and the determination of the energy of the first excited $2^{+}$state in ${ }^{78,80} \mathrm{Zn}$, which have already been reported in Ref. [14]. Furthermore, $B\left(E 2,4_{1}^{+} \rightarrow 2_{1}^{+}\right)$values in ${ }^{74,76} \mathrm{Zn}$ are reported in addition. The production method, the postacceleration at REX-ISOLDE, and the normalization of the different experiments are extensively described in Sec. II. In Sec. III, the extended zinc systematics are compared to newly performed shell-model calculations, collective model predictions, and systematics of neighboring even-even isotopes.

\section{MEASUREMENTS}

\section{A. Production, postacceleration, and Coulomb excitation of the radioactive ion beams}

The zinc beams were produced by bombarding a $50 \mathrm{~g} / \mathrm{cm}^{2}$ $\mathrm{UC}_{x}$ target, enriched in ${ }^{238} \mathrm{U}$, with $1.4-\mathrm{GeV}$ protons at a
TABLE I. Characteristics of the zinc radioactive ion beams. Columns 1 and 2 give the mass and half-life, respectively, of the considered zinc isotope (taken from Ref. [20]). The fraction of gallium over zinc isotopes in the laser-ionized postaccelerated beam is given in the third column. The measured beam intensity of zinc isotopes after mass separation (1) and postacceleration (2) is given in columns 4 and 5. $\epsilon_{\mathrm{REX}}$ refers to the overall transmission efficiency from the primary to the secondary target (see text for details).

\begin{tabular}{cccccc}
\hline \hline$A$ & $T_{1 / 2}$ & $f_{\mathrm{Ga}} / f_{\mathrm{Zn}}$ & \multicolumn{2}{c}{ Zinc beam intensity (ions/s) } & \multirow{2}{*}{$\epsilon_{\mathrm{REX}}$} \\
& $(\mathrm{s})$ & $(\%)$ & $(1)$ & $(2)$ & \\
\hline 74 & $95.6(12)$ & 0.1 & $4.0 \mathrm{E} 7$ & $3.0 \mathrm{E} 5$ & $\sim 1 \%$ \\
76 & $5.7(3)$ & $1.5(1)$ & $1.0 \mathrm{E} 7$ & $1.1 \mathrm{E} 5$ & $\sim 1 \%$ \\
78 & $1.47(15)$ & $5.8(6)$ & $7.8 \mathrm{E} 5$ & $4.3 \mathrm{E} 3$ & $\sim 1 \%$ \\
80 & $0.54(2)$ & $16.2(6)$ & $6.8 \mathrm{E} 4$ & $3.0 \mathrm{E} 3$ & $\sim 4-5 \%$ \\
\hline \hline
\end{tabular}

maximum intensity of $3.2 \times 10^{13}$ protons/pulse and an average pulse repetition rate of $2.4 \mathrm{~s}$, delivered by the PS Booster at CERN. The isotopes produced diffuse out of the heated primary target through a transfer line toward another heated tube, the ionizer cavity. Laser light is sent inside this ionizer cavity and zinc isotopes are resonantly ionized. The $1^{+}$charged zinc ions are extracted from the ion source by applying a $60-\mathrm{kV}$ electrostatic potential and are subsequently mass separated by the General Purpose Separator (GPS). The selectivity of the RILIS (Resonance Ionization Laser Ion Source) [15] is hampered by the high temperature of the ion source cavity, because elements with a low ionization potential are surface ionized. The surface-ionized species are extracted as well and result in isobaric contamination after mass separation. The elements with low ionization potentials (IP) near zinc $(Z=30$, $\mathrm{IP}=9.4 \mathrm{eV})$ are gallium $(Z=31, \mathrm{IP}=6.0 \mathrm{eV})$ and rubidium $(Z=37, \mathrm{IP}=4.2 \mathrm{eV})$ [16]. Thus, isobaric contamination is expected from gallium and rubidium isotopes. The average beam intensities of the investigated zinc isotopes after mass separation are given in Table I.

The isobaric beam, consisting of laser- and surface-ionized isotopes, is continuously injected in REXTRAP [17], where the incoming ions are trapped for a time ranging from 0 to $79 \mathrm{~ms}$, depending on the time when the ion was injected. During this trapping time, the ions are cooled by collisions with buffer gas atoms (natural $\mathrm{Ar} / \mathrm{Ne}$ gas). After this cooling period, the ions are efficiently transmitted as a bunch to the Electron Beam Ion Source (EBIS) [18], which operates at the same repetition rate as REXTRAP $(12.7 \mathrm{~Hz})$. All ions spent an equal amount of time in the EBIS, during which they are brought to high charge states. The ions are extracted from the EBIS in a pulse of $\approx 700 \mu \mathrm{s}$ and are separated according to their $A / q$ ratio, prior to injection in the REX linear accelerator. The highly charged ions $\left(q=20^{+}\right.$for $A=74,76$ and $q=21^{+}$for $A=78,80)$ were postaccelerated by the REX linear accelerator [19] to a final energy of $2.87 \mathrm{MeV} /$ nucleon $(A=74,78)$, $2.83 \mathrm{MeV} /$ nucleon $(A=76)$, and $2.79 \mathrm{MeV} /$ nucleon $(A=80)$.

A major constraint on the possible isobaric contaminants in the beam is given by the chemical selectivity of the laser ion source $[15,21,22]$ and the in-target production cross 

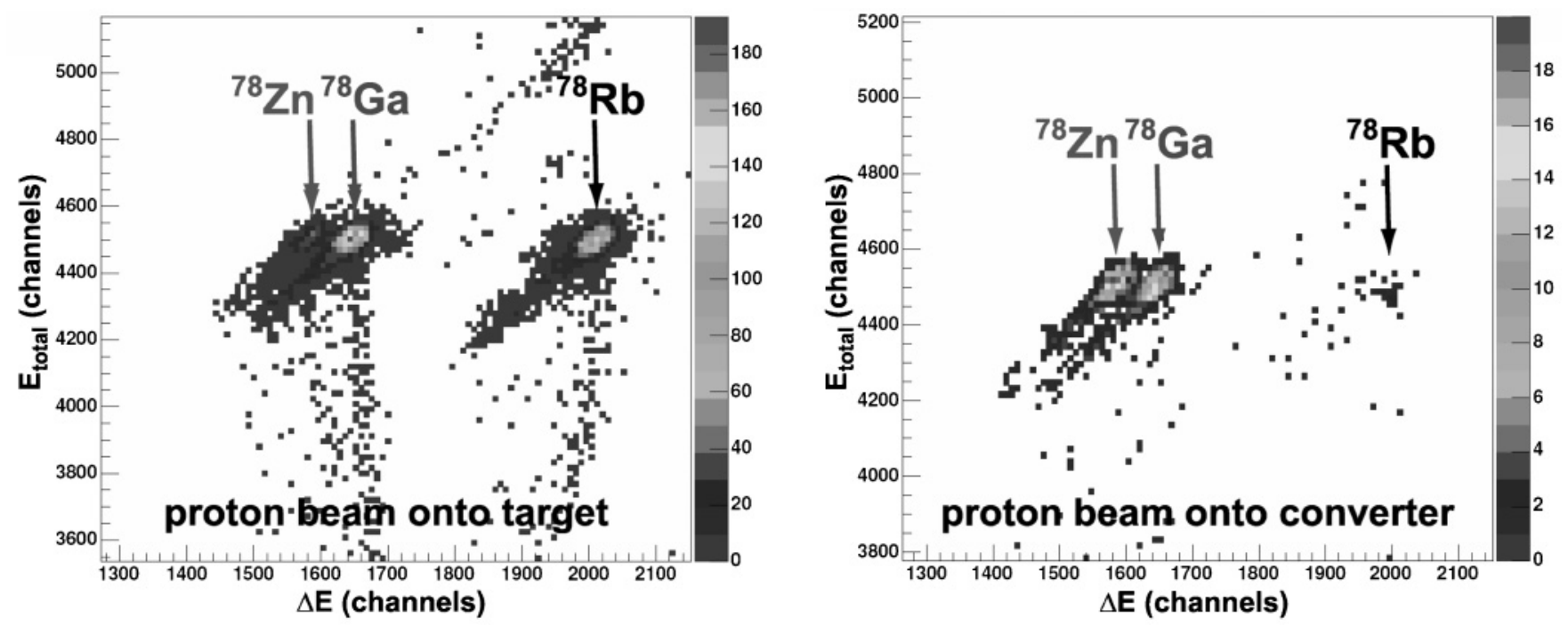

FIG. 1. $\Delta E$-E identification plot of the $A=78$ postaccelerated beam. (Left) Protons directly on the $\mathrm{UC}_{x}$ target (high-energy proton-induced fission of uranium); (right) protons on the neutron converter (neutron-induced fission of uranium).

sections. Measured production cross sections of ${ }^{74,76} \mathrm{Rb}$ in proton-induced fission of uranium at $1 \mathrm{GeV} /$ nucleon in inverse kinematics [23] are an order of magnitude lower than for ${ }^{74,76} \mathrm{Zn}$ and ${ }^{74,76} \mathrm{Ga}$. Indeed, no evidence was found for $\gamma$ rays from ${ }^{76} \mathrm{Rb}$ or daughter decay products from ${ }^{74} \mathrm{Rb}$ (because no $\gamma$ rays are known in the $\beta$ decay of ${ }^{74} \mathrm{Rb}$ ), in the measured $\beta$-decay $\gamma$ spectrum observed in the MINIBALL array at the secondary target position and in the single coaxial germanium detector positioned near the beam dump.

The production cross section for ${ }^{78} \mathrm{Rb}$ in high-energy proton-induced fission of uranium attains the same order of magnitude as ${ }^{78} \mathrm{Zn}$ [23]. The $A=78$ beam was sent into a $\triangle E-E$ (gas-Si) telescope, installed at the end of the linear accelerator to check the beam composition. The ions were identified in a $\Delta E-E$ plot, which is shown in Fig. 1. A strong ${ }^{78} \mathrm{Rb}$ contamination is observed when the $A=78$ beam is produced by high-energy proton-induced fission of uranium (Fig. 1, left). To suppress the rubidium contamination, the proton beam was directed on a heavy metal (W) rod (the "neutron converter"), resulting in a flux of spallation neutrons escaping from the metal rod. These induce mainly fission in the neighboring $\mathrm{UC}_{x}$ primary target [24]. The $\Delta E-E$ identification plot (Fig. 1, right) shows that the ${ }^{78} \mathrm{Rb}$ contamination is largely suppressed when the ions are produced by neutron-induced fission.

During the $A=80$ experiment, the primary target was equipped with a quartz transfer line [25], which absorbs a major part of the largely produced and surface ionized ${ }^{80} \mathrm{Rb}$. Radioactivity from ${ }^{80} \mathrm{Ga}$ and ${ }^{80} \mathrm{Rb}$ was identified in the $\beta$-decay $\gamma$ spectrum at the secondary target position and in the beam dump. From the intensity balance of $\beta$-decay $\gamma$ rays from ${ }^{80} \mathrm{Ga}$ and its decay daughter products, no evidence was found for other isobaric contaminants. The high chemical selectivity of the quartz transfer line, which was available only during the $A=80$ experiment, allowed use of high-energy proton-induced fission that gives about three time higher yields for the considered zinc isotopes compared to the usage of the converter and neutron-induced fission [26].

An additional source of isobaric beam contamination becomes apparent when the half-life of the investigated isotope is comparable to the trapping and charge breeding time. In those cases, decay losses of the short-lived isotope in the REXTRAP and EBIS need to be taken into account. The decay of zinc isotopes into gallium isotopes during the bunching and charge breeding periods adds to the gallium contamination from the surface ionization in the primary target. The fraction of zinc isotopes that decays to radioactive gallium isotopes during the trapping time was calculated analytically, assuming a constant release from the primary target during the 79-ms trapping period and a constant charge breeding time for all isotopes. The fraction of zinc or gallium isotopes in the postaccelerated, laser ionized beam is denoted $f_{\mathrm{Zn}}$ or $f_{\mathrm{Ga}}$, respectively, and the ratios $f_{\mathrm{Ga}} / f_{\mathrm{Zn}}$ are given in Table I for all considered masses. The considered gallium isotopes are sufficiently long lived to ensure that $f_{\mathrm{Ga}}+f_{\mathrm{Zn}} \approx 1$ and the production of grand-daughter isotopes is thus not considered.

The postaccelerated radioactive ion beam was sent on a secondary thin target, where Coulomb excitation was induced on all isobaric isotopes. Typical average zinc beam intensities on the secondary target are summarized in Table I. The REX transmission efficiency of $1-5 \%$ includes the trapping efficiency, charge breeding efficiency for the selected $A / q$ and the overall transport efficiency of the accelerator. For the $A=74,76$ beams, a $2.3-\mathrm{mg} / \mathrm{cm}^{2}{ }^{120} \mathrm{Sn}$ target was used. The moderately low-excitation cross-section and the high $E\left(2_{1}^{+}\right)$of ${ }^{120} \mathrm{Sn}$ make this target well suited for relatively higher intensity $A=74,76$ beams. For the lower intensity beams of $A=78,80$ a $2.0-\mathrm{mg} / \mathrm{cm}^{2}{ }^{108} \mathrm{Pd}$ target was chosen with a higher excitation probability and with de-excitation lines that would not contaminate the region where the $2_{1}^{+}$ states of ${ }^{78,80} \mathrm{Zn}$ were expected to occur. The secondary target was surrounded by the MINIBALL germanium detector array, consisting of eight cluster detectors [27], to detect the 
prompt $\gamma$ rays following the Coulomb excitation. Each cluster detector consists of three germanium crystals, which are in turn electrically segmented into six parts. The high segmentation of the MINIBALL array allows the determination of the angular coordinates of the detected $\gamma$ rays accurately, relative to the center of the detector array. The absolute full-energy peak efficiency of the detector array is $7.7 \%$ at $1.173 \mathrm{MeV}$ after cluster add-back. The scattered beam nuclei and the recoiling target nuclei are detected with a double-sided silicon strip detector (DSSSD) [28]. The DSSSD covers an angular range from $16.4^{\circ}$ to $53.4^{\circ}$ in the laboratory. For the $A=74,76,78$ experiments, the active area of the DSSSD was limited to an angular range from $29.1^{\circ}$ to $53.4^{\circ}$ to prevent radiation damage to the inner rings of the detector due to the high elastic scattering rate and the relatively high beam intensity for $A=74,76$. The front of the detector is segmented in 16 annular strips, providing information on the laboratory scattering angle $\theta$ of the particles. The back side is segmented in 24 sectors, providing the azimuthal scattering angle. Detected recoiling target and scattered beam particles could be separated in the DSSSD, based on their different kinematics. The angular information of the detected particle and $\gamma$ ray is used to perform a Doppler correction of the $\gamma$-ray energy on an event-by-event basis, assuming the $\gamma$ ray was emitted in-flight by the detected particle. Over the detected angular scattering ranges, the distance of closest approach $(d)$ fulfills the condition $d>1.25\left(A_{P}^{1 / 3}+A_{T}^{1 / 3}\right)+5 \mathrm{fm}$, ensuring that the excitation is purely electromagnetic [29].

\section{B. Data reduction and analysis}

Data was acquired during $800 \mu \mathrm{s}$, covering the arrival time of the particles at the secondary target after they have been ejected from the EBIS. These spectra will be referred to as inbeam singles $\gamma$-ray spectra. In between two beam pulses from the EBIS, data were acquired during the same amount of time $(800 \mu \mathrm{s})$, which will be referred to as off-beam singles $\gamma$-ray spectra. The $\gamma$ rays, detected with the MINIBALL array, were registered during these time periods without any conditioning. The unconditioned in-beam singles $\gamma$-ray spectra contain the room background, the $\beta$-decay activity, originating from scattered beam particles in the target chamber, $\mathrm{x}$ rays from the accelerator cavities, and prompt radiation following the Coulomb excitation process. The off-beam spectra do contain neither beam- nor accelerator-related radiation, because the REX linear accelerator is pulsed at the same rate as the EBIS. The in-beam singles $\gamma$-ray spectrum is shown in Fig. 2(a) for the $A=74$ experiment. As indicated above, the high statistics singles $\gamma$-ray spectra were used to identify different radioactive contaminants in the isobaric ion beam. To suppress the dead time in the particle detector electronics during the $A=74,76$ experiments, the particle trigger consisted of a particle- $\gamma$ coincidence trigger (600-ns coincidence window) and a particle trigger that was downscaled by a factor $2^{6}$. The $\gamma$ rays within a prompt time coincidence window of $225 \mathrm{~ns}$ were selected to construct the Coulomb excitation spectra. The prompt particle- $\gamma$ time coincidence window is shown in Fig. 2(d) in black and the resulting "prompt" $\gamma$-ray spectrum

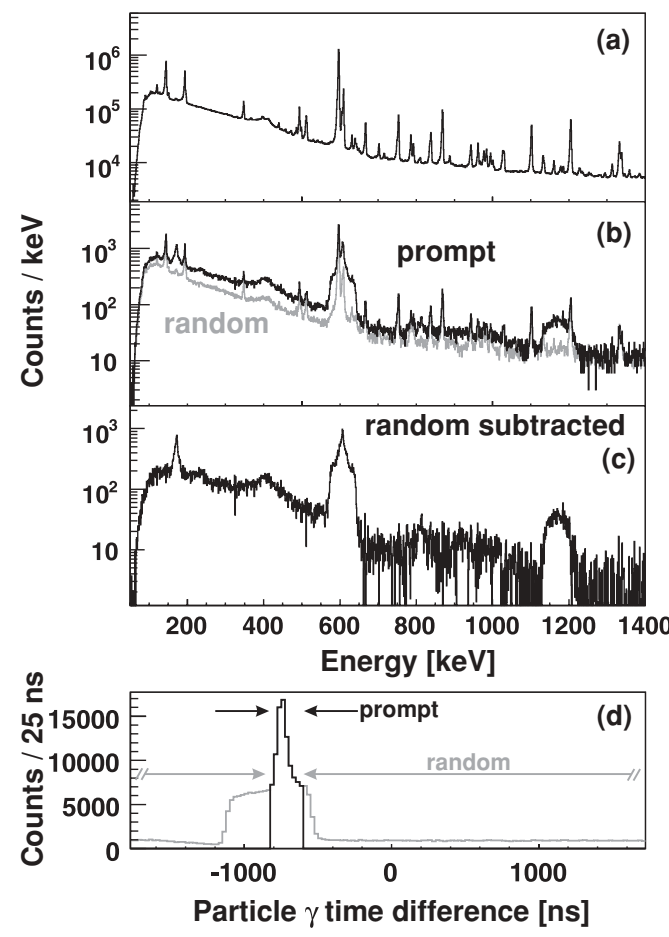

FIG. 2. (a) In-beam singles $\gamma$-ray spectrum observed in the MINIBALL array. (b) $\gamma$-ray spectrum in prompt (black) and random (red) coincidence with detected beam and/or target particles in the DSSSD. The random spectra is scaled with a factor 0.31 (see text for details). (c) Random subtracted prompt $\gamma$-ray spectrum for $A=74$. (d) Prompt and random coincident time windows.

is shown in Fig. 2(b). A huge reduction of the radioactive $\beta$-decay background is obtained. Random coincidences were selected over a particle- $\gamma$ time difference window of in total $3.75 \mu \mathrm{s}$, which is partly shown in Fig. 2(d). Appropriate scaling factors for the random spectra were obtained from the average intensity ratio of $\beta$-decay lines observed in both random and prompt spectra. These scaling factors were $0.31(0.33,0.12,0.48)$ for the $A=74(76,78,80)$ experiments. The resulting scaled "random" $\gamma$-ray spectrum for $A=74$ is shown in Fig. 2(b) in gray. The random subtracted prompt $\gamma$-ray spectrum is shown in Fig. 2(c) for $A=74$ and a background-free Coulomb excitation spectrum is obtained, where the different Coulomb excitation lines can be integrated reliably.

During the experiments, the selective laser ionization (see Sec. II A) was periodically turned ON and OFF to normalize the experiments (see Sec. II C for more details). Figures 3, 4,5 , and 6 show the resulting Coulomb excitation spectra acquired during the $A=74,76,78,80$ experiments after random subtraction. The $\gamma$-ray spectra labeled (a) are observed in prompt coincidence with detected recoiling target particles during laser ON periods. The detected $\gamma$-ray energies are Doppler corrected for the in-flight decaying target nuclei. The spectra labeled (b) are observed in prompt coincidence with detected scattered beam particles during laser $\mathrm{ON}$ periods. The detected $\gamma$-ray energies are Doppler corrected for the in-flight decaying beam nuclei. The spectra labeled (c) are conditioned 


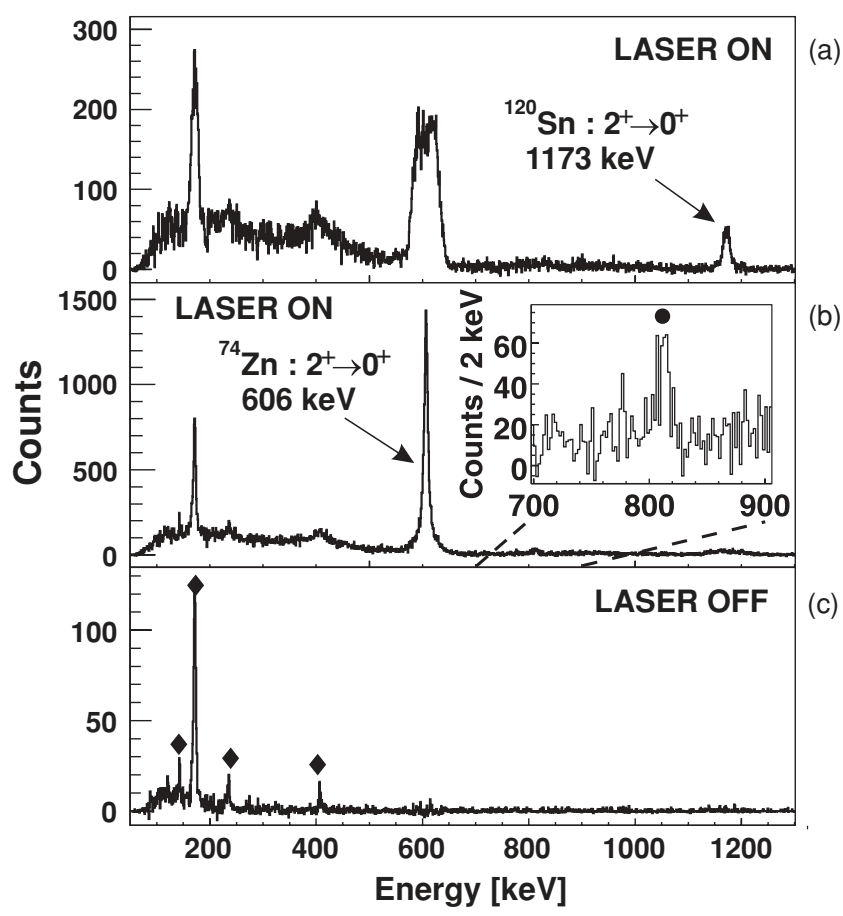

FIG. 3. Coulomb excitation spectra for the $A=74$ beam: (a) prompt coincident with a recoiling target particle, random subtracted and Doppler corrected for the de-exciting target nucleus, during laser ON periods; (b) prompt coincident with a scattered beam particle, random subtracted and Doppler corrected for the de-exciting beam nucleus, during laser ON periods. The filled circle indicates the $4_{1}^{+} \rightarrow$ $2_{1}^{+}$transition in ${ }^{74} \mathrm{Zn}$ at $813 \mathrm{keV}$; (c) same as (b) but during laser OFF periods. The several de-excitation $\gamma$ rays from the target and zinc nuclei are indicated with text labels, de-excitation lines from the isobaric contaminant are indicated with diamonds.

in the same way as the spectra labeled (b) but were acquired during laser OFF periods.

In Figs. 3(a) and 4(a), the $2_{1}^{+} \rightarrow 0_{1}^{+}$transition at $1173 \mathrm{keV}$ in the target nucleus ${ }^{120} \mathrm{Sn}$ is clearly observed after Doppler correction. In Figs. 5(a) and 6(a), the $2_{1}^{+} \rightarrow 0_{1}^{+}$transition at $434 \mathrm{keV}$ in the target nucleus ${ }^{108} \mathrm{Pd}$ is clearly observed as well with high statistics in both cases. Also $\gamma$ rays de-exciting higher-lying states in ${ }^{108} \mathrm{Pd}$ are observed in both Figs. 5 and 6: $497 \mathrm{keV}\left(2_{2}^{+} \rightarrow 2_{1}^{+}\right), 614 \mathrm{keV}\left(4_{1}^{+} \rightarrow 2_{1}^{+}\right)$, $619 \mathrm{keV}\left(0_{2}^{+} \rightarrow 2_{1}^{+}\right)$, and $931 \mathrm{keV}\left(2_{2}^{+} \rightarrow 0_{1}^{+}\right)$.

In the $A=74$ laser OFF spectrum [Fig. 3(c)], four Coulomb excitation lines are visible from the isobaric contaminant $\left({ }^{74} \mathrm{Ga}\right)$ at $143,171,235$, and $407 \mathrm{keV}$, where only the $171-\mathrm{keV}$ line can be accounted for by the known level scheme of ${ }^{74} \mathrm{Ga}$. Two intense Doppler-corrected transitions from the isobaric contaminant ${ }^{76} \mathrm{Ga}$ are observed at 160 and $254 \mathrm{keV}$ in the $A=76$ laser OFF spectrum [Fig. 4(c)]. Neither of these $\gamma$ rays can be placed in the known level scheme of ${ }^{76} \mathrm{Ga}$. In the $A=78$ laser OFF spectra [Fig. 5(c)], one low-energy transition is observed at $280 \mathrm{keV}$, which de-excites a known level at $280 \mathrm{keV}$ in ${ }^{78} \mathrm{Ga}$. Four transitions are observed in the $A=80$ laser OFF spectrum [Fig. 6(c)]. Three $\gamma$ rays (175, 201 , and $376 \mathrm{keV}$ ) can be placed in the known level scheme of ${ }^{80} \mathrm{Rb}$. One $\gamma$ ray at $889 \mathrm{keV}$ de-excites a known energy level in ${ }^{80} \mathrm{Ga}$. These laser OFF spectra provide additional confidence

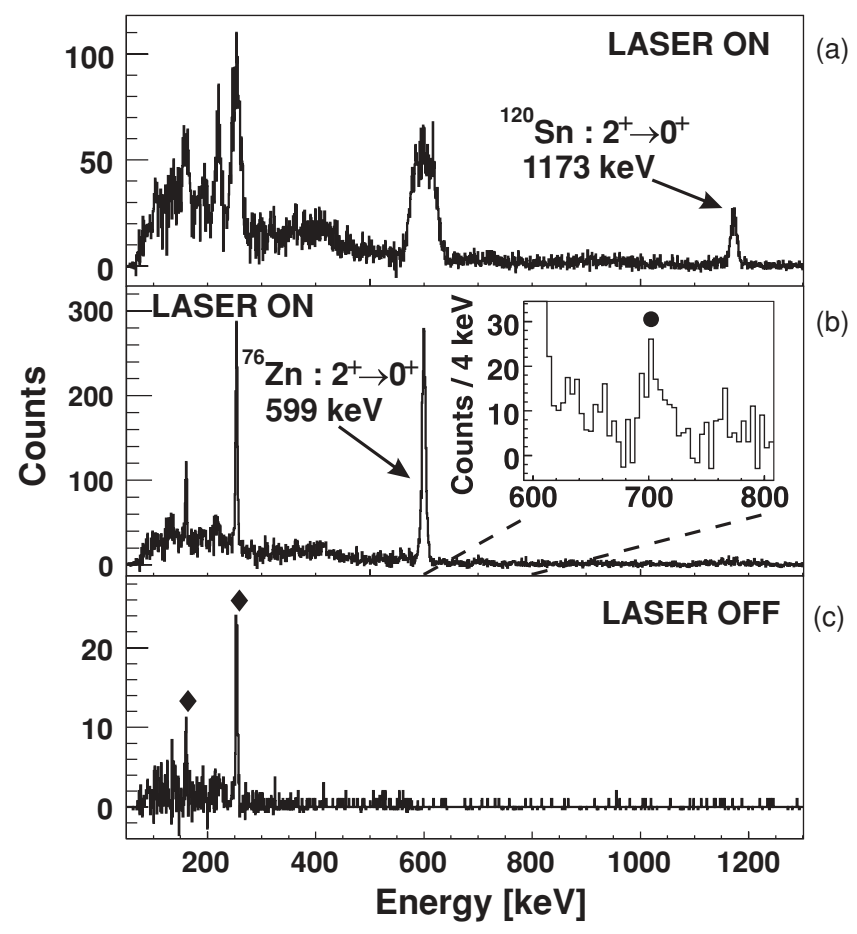

FIG. 4. Similar spectra as in Fig. 3 for the $A=76$ experiment. The filled circle in (b) indicates the $4_{1}^{+} \rightarrow 2_{1}^{+}$transition in ${ }^{76} \mathrm{Zn}$ at $697 \mathrm{keV}$.

in the identification of the different isobaric contaminants in the radioactive ion beams.

By comparing the laser ON (b) and laser OFF (c) Dopplercorrected spectra, the transitions belonging to zinc isotopes are clearly identified. The $2_{1}^{+} \rightarrow 0_{1}^{+}$transition at $606 \mathrm{keV}$ and the $4_{1}^{+} \rightarrow 2_{1}^{+}$transition at $813 \mathrm{keV}$ in ${ }^{74} \mathrm{Zn}$ are clearly identified in Fig. 3(b) and the $2_{1}^{+} \rightarrow 0_{1}^{+}$transition at $599 \mathrm{keV}$ and a weak trace of the $4_{1}^{+} \rightarrow 2_{1}^{+}$transition at $697 \mathrm{keV}$ in ${ }^{76} \mathrm{Zn}$ are identified in Fig. 4(b). A 730-keV $\gamma$ ray was observed before in an isomeric decay study of the $8_{1}^{+}$state in ${ }^{78} \mathrm{Zn}[30]$ and in a $\beta$-decay study at ISOLDE [31] and it was tentatively identified as the $2_{1}^{+}$state in ${ }^{78} \mathrm{Zn}$. Because in Coulomb excitation, the dominant excitation cross section is of $E 2$ type, the spin and parity of the $730-\mathrm{keV}$ level is now established, as evidenced in Fig. 5(b). The $2_{1}^{+}$state in ${ }^{80} \mathrm{Zn}$ has not been observed before and this Coulomb excitation study provides the first observation of this state at $1492 \mathrm{keV}$.

The Coulomb excitation cross sections and the $\gamma$-ray yields following the de-excitation process were calculated with the computer code GOSIA2, which is based on the electromagnetic excitation theory by Alder and Winther [32-34]. GOSIA2 takes into account the angular distribution of the $\gamma$ rays, the energy loss of the beam in the target, the Doppler shift of the detected $\gamma$-ray energy in each $\gamma$ detector, and the relative efficiency curve of each $\gamma$ detector. The $\gamma$-ray yield from the beam nuclei (zinc isotopes) was normalized to the observed $\gamma$-ray yield from the target nuclei. The calculated ratio of $2_{1}^{+} \rightarrow 0_{1}^{+}$ yield from target and zinc nuclei was fitted to the experimental ratio by changing the $B(E 2 \downarrow)\left(=\frac{1}{5}\left|\left\langle 0_{1}^{+}|| E 2|| 2_{1}^{+}\right\rangle\right|^{2}\right)$ value for the zinc isotopes in GOSIA2, while the $2_{1}^{+}$quadrupole moment $\left(=0.7579\left\langle 2_{1}^{+} \| E 2|| 2_{1}^{+}\right\rangle\right)$was fixed to $0 e \mathrm{~b}$ for all zinc isotopes. 
TABLE II. Overview of the experimental results for ${ }^{74,76,78,80} \mathrm{Zn}$ and the total measuring time on each mass with the laser ON, in laser ON/OFF mode, and with the laser OFF.

\begin{tabular}{|c|c|c|c|c|c|c|c|c|c|c|}
\hline \multirow[t]{2}{*}{ A } & \multicolumn{2}{|c|}{$\mathrm{Zn} /$ Total $(\%)$} & $B\left(E 2,2_{1}^{+} \rightarrow 0_{1}^{+}\right)$ & \multirow[t]{2}{*}{$B\left(E 2,4_{1}^{+} \rightarrow 2_{1}^{+}\right)$} & \multirow{2}{*}{$\begin{array}{c}\tau_{2_{1}^{+}} \\
\text {(ps) }\end{array}$} & \multirow{2}{*}{$\begin{array}{l}E\left(2_{1}^{+}\right) \\
(\mathrm{keV})\end{array}$} & \multirow{2}{*}{$\begin{array}{l}E\left(4_{1}^{+}\right) \\
(\mathrm{keV})\end{array}$} & \multicolumn{3}{|c|}{ Measuring time } \\
\hline & $\mathrm{ON} / \mathrm{OFF}$ & $O N$ & $\left(e^{2} \mathrm{fr}\right.$ & & & & & $\mathrm{ON}$ & ON/OFF & OFF \\
\hline 74 & $82(1)$ & $83(4)$ & $401(32)$ & $507(74)$ & $24.8(20)$ & 606 & 813 & $8.5 \mathrm{~h}$ & $3 \mathrm{~h}$ & - \\
\hline 76 & $79(2)$ & $73(7)$ & $290(36)$ & $320(91)$ & $36.4(46)$ & 599 & 697 & $11.5 \mathrm{~h}$ & $2.5 \mathrm{~h}$ & - \\
\hline 78 & $65(2)$ & $64(13)$ & $154(38)$ & - & $25.5(61)$ & 730 & $(891)$ & $23 \mathrm{~h}$ & $9 \mathrm{~h}$ & - \\
\hline \multirow[t]{4}{*}{80} & $61(5)^{\mathrm{a}}$ & - & $150(32)$ & - & $0.74(16)$ & 1492 & - & $102 \mathrm{~h}$ & $6.5 \mathrm{~h}$ & $6.5 \mathrm{~h}$ \\
\hline & $44(5)^{\mathrm{b}}$ & - & $144(29)$ & - & $0.77(16)$ & & & & & \\
\hline & - & $61(6)^{a}$ & $151(37)$ & - & $0.73(18)$ & & & & & \\
\hline & - & $46(9)^{b}$ & $138(43)$ & - & $0.80(25)$ & & & & & \\
\hline
\end{tabular}

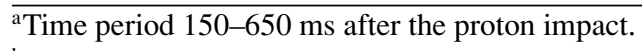

${ }^{\mathrm{b}}$ Time period 0-2400 $\mathrm{ms}$ after the proton impact.

The resulting $B(E 2 \downarrow)$ values are summarized in Table II. In Sec. II D, the influence of a nonzero quadrupole moment and virtual excitation of higher-lying states $\left(4_{1}^{+}\right.$and $\left.2_{2}^{+}\right)$will be discussed. The matrix elements connecting all relevant states in the excitation process of the target nuclei were deduced from $B(E 2 \downarrow)$ and $Q_{2_{1}^{+}}$literature values, taken from Refs. [35,36] for ${ }^{120} \mathrm{Sn}$ and from Refs. [20,37] for ${ }^{108} \mathrm{Pd}$.

\section{Beam contamination and normalization}

The determination of the $B\left(E 2,2_{1}^{+} \rightarrow 0_{1}^{+}\right)$values depends strongly on the knowledge of the experimental $\gamma$-ray yield from the target nucleus that was induced by incoming zinc isotopes. Thus, the beam composition is a crucial parameter in the normalization to the target excitation. As discussed in Sec. II A, the composition of the beam before injection in the

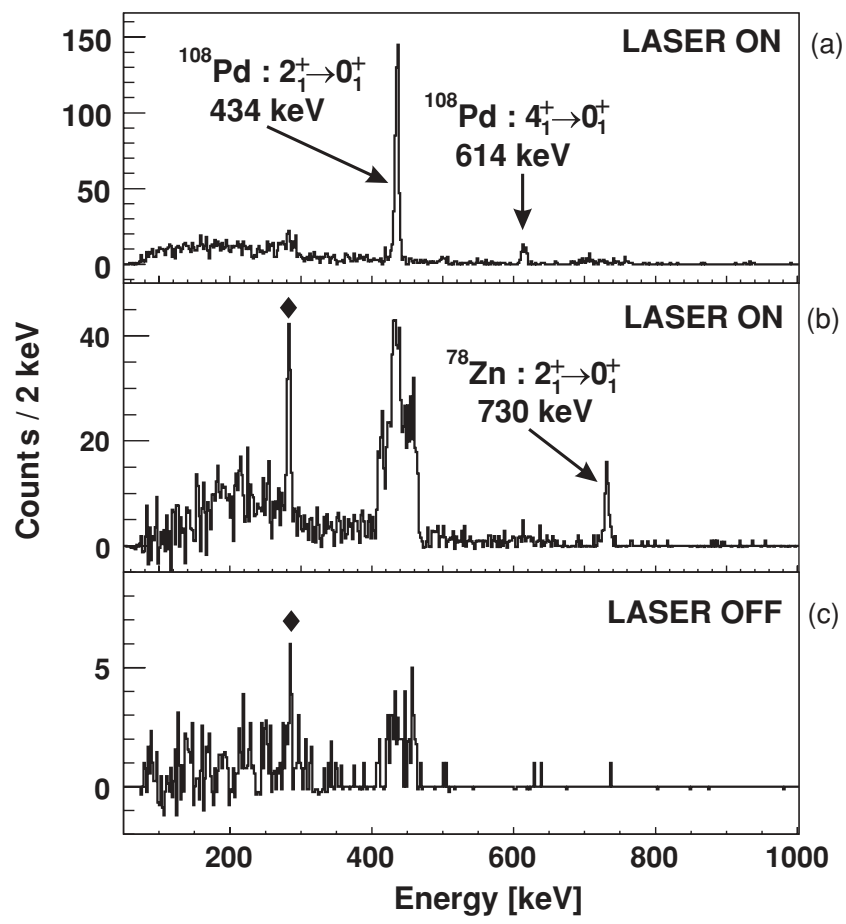

FIG. 5. Similar spectra as in Fig. 3 for the $A=78$ experiment.
REX-TRAP is different than the beam extracted from REXEBIS due to the decay of zinc to gallium isotopes during the trapping and charge-breeding processes. Taking $I^{l}$ as the total beam intensity due to laser ionization, this intensity can be written as a mixture of zinc and gallium isotopes: $f_{\mathrm{Zn}} I^{l}+$ $f_{\mathrm{Ga}} I^{l}$, taking the $f$ values from Table $\mathrm{I}$ and using the fact that $f_{\mathrm{Ga}}+f_{\mathrm{Zn}} \approx 1$. The number of target de-excitation $\gamma$ rays induced by zinc isotopes is given by

$N_{\gamma, T}^{\mathrm{Zn}}=N_{\gamma, T}^{\mathrm{tot}} \times\left[1+\frac{f_{\mathrm{Ga}}}{f_{\mathrm{Zn}}} \times \frac{\sigma_{T}^{\mathrm{Ga}}}{\sigma_{T}^{\mathrm{Zn}}}+\sum_{x} \frac{I_{x}^{s}}{f_{\mathrm{Zn}} I^{l}} \times \frac{\sigma_{T}^{x}}{\sigma_{T}^{\mathrm{Zn}}}\right]^{-1}$,

where $I_{x}^{s}$ is the surface ionized beam intensity from isotope $x$ and $\sigma_{T}^{x}$ is the total Coulomb excitation cross section for

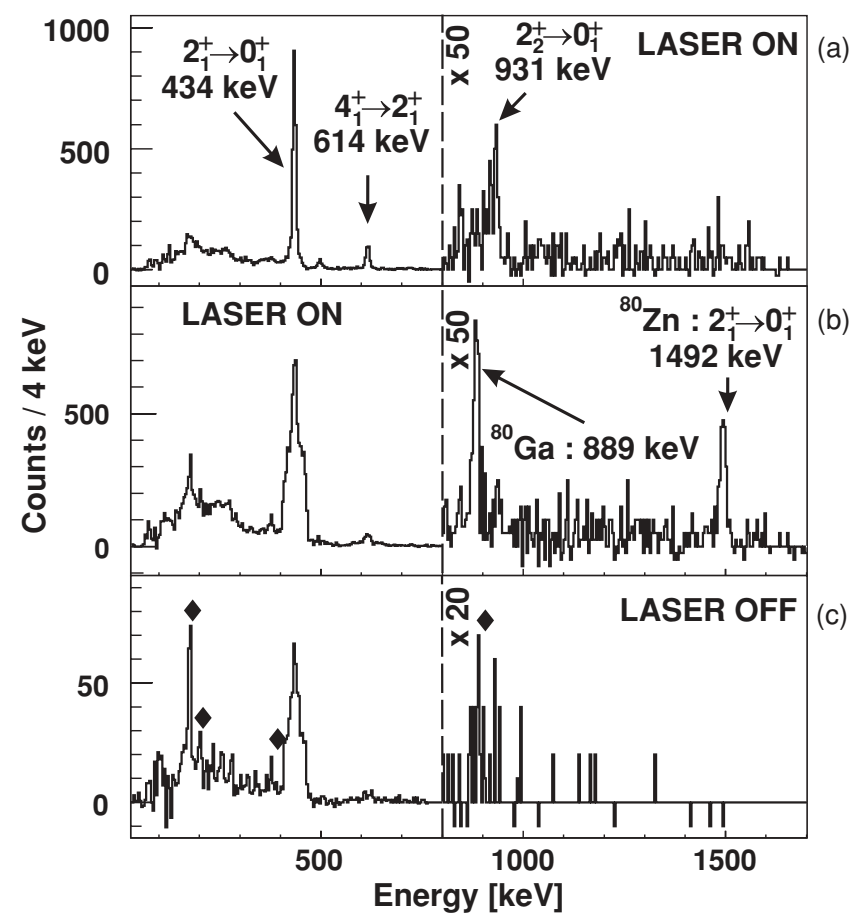

FIG. 6. Similar spectra as in Fig. 3 for the $A=80$ experiment. 
the $2_{1}^{+} \rightarrow 0_{1}^{+}$transition in the target nucleus $(T)$ when it was excited by the isotope $x$.

$$
\text { 1. }{ }^{74,76,78} \mathrm{Zn}
$$

As discussed above, the only isobaric contamination in the $A=74,76,78$ beams originates from surface ionized gallium $(Z=31)$, so Eq. (1) can be reduced to

$$
N_{\gamma, T}^{\mathrm{Zn}}=N_{T}^{\text {Total }} \times\left[1+\left(\frac{f_{\mathrm{Ga}}}{f_{\mathrm{Zn}}}+\frac{I_{\mathrm{Ga}}^{s}}{f_{\mathrm{Zn}} I^{l}}\right) \times \frac{\sigma_{T}^{\mathrm{Ga}}}{\sigma_{T}^{\mathrm{Zn}}}\right]^{-1},
$$

where $I_{\mathrm{Ga}}^{s}$ is the surface ionized gallium beam intensity. The ratio of surface-ionized gallium over laser-ionized zinc isotopes in the beam $\left(\frac{I_{\mathrm{Ga}}^{s}}{f_{\mathrm{zn}} I^{I}}\right)$ was determined by switching the laser ionization periodically on and off with a typical periodicity of $\sim 20 \mathrm{~s}$. Averaged over a the complete laser ON/OFF run, the laser is blocked for $50 \%$ of the time. These "laser ON/OFF" runs were performed at regular time intervals during the experiments and are shown with the shaded areas in Fig. 7. In total, $25 \%(22 \%, 29 \%)$ of the measuring time on mass $A=74(76,78)$ was spent in this laser ON/OFF mode. From the number of scattered particles in the DSSSD during laser ON $\left(\mathrm{N}_{\mathrm{ON}}\right)$ and laser OFF $\left(\mathrm{N}_{\mathrm{OFF}}\right)$ periods, the ratio $\frac{I_{\mathrm{Ga}}^{s}}{f_{\mathrm{Zn}} I^{I}}$ can be determined as follows:

$$
\left(\frac{I_{\mathrm{Ga}}^{s}}{f_{\mathrm{Zn}} I^{l}}\right)=\left(\frac{f_{\mathrm{Ga}}}{f_{\mathrm{Zn}}}+\frac{\sigma_{R}^{\mathrm{Zn}}}{\sigma_{R}^{\mathrm{Ga}}}\right) \times \frac{N_{\mathrm{OFF}}}{N_{\mathrm{ON}}-N_{\mathrm{OFF}}},
$$

where $\sigma_{R}^{x}$ denotes the elastic Rutherford cross section for element $x$. Possible fluctuations in the laser ionization efficiency during laser ON periods can change the ratio $\frac{I_{\mathrm{Ga}}^{S}}{f_{\mathrm{Zn}} I^{i}}$ and thus influence the final result. To have a reliable measure of the beam contamination during laser ON periods, the ratio measured during laser ON/OFF periods was related to prompt observed gallium and zinc de-excitation $\gamma$ rays $\left(\mathrm{N}_{\gamma, \mathrm{Ga}}\right.$ and $\mathbf{N}_{\gamma, \mathrm{Zn}}$, respectively), which are directly proportional to the gallium and zinc content in the beam. The $171-\mathrm{keV}$ and 254-keV lines related to ${ }^{74} \mathrm{Ga}$ and ${ }^{76} \mathrm{Ga}$ were used together with the 606- and 599-keV $2_{1}^{+} \rightarrow 0_{1}^{+}$lines from ${ }^{74} \mathrm{Zn}$ and ${ }^{76} \mathrm{Zn}$. This results in the following relation between $\frac{I_{\mathrm{Ga}}^{s}}{f_{\mathrm{Zn}^{\mathrm{n}}} I^{i}}$ and $\mathbf{N}_{\gamma, \mathrm{Zn} / \mathrm{Ga}}$ during laser ON/OFF periods:

$$
\kappa_{1}=\left(\frac{f_{\mathrm{Ga}}}{f_{\mathrm{Zn}}}+2 \times \frac{I_{\mathrm{Ga}}^{s}}{f_{\mathrm{Zn}} I^{l}}\right) \times \frac{N_{\gamma, \mathrm{Zn}}}{N_{\gamma, \mathrm{Ga}}},
$$

where the factor 2 stems from the fact that half of the measuring time, the $\mathrm{Zn}$ beam was not present. The ratio $\frac{I_{\mathrm{Gi}}^{s}}{f_{\mathrm{Zn}} I^{l}}$ during laser ON periods can be extracted based on the observed gallium and zinc de-excitation $\gamma$ rays:

$$
\left(\frac{I_{\mathrm{Ga}}^{s}}{f_{\mathrm{Zn}} I^{l}}\right)^{\mathrm{ON}}=\frac{N_{\gamma, \mathrm{Ga}}}{N_{\gamma, \mathrm{Zn}}} \times \kappa_{1}-\frac{f_{\mathrm{Ga}}}{f_{\mathrm{Zn}}}
$$

where the factor 2 has been omitted, because the laser ionization (and thus the zinc beam) was present during the full measuring time.
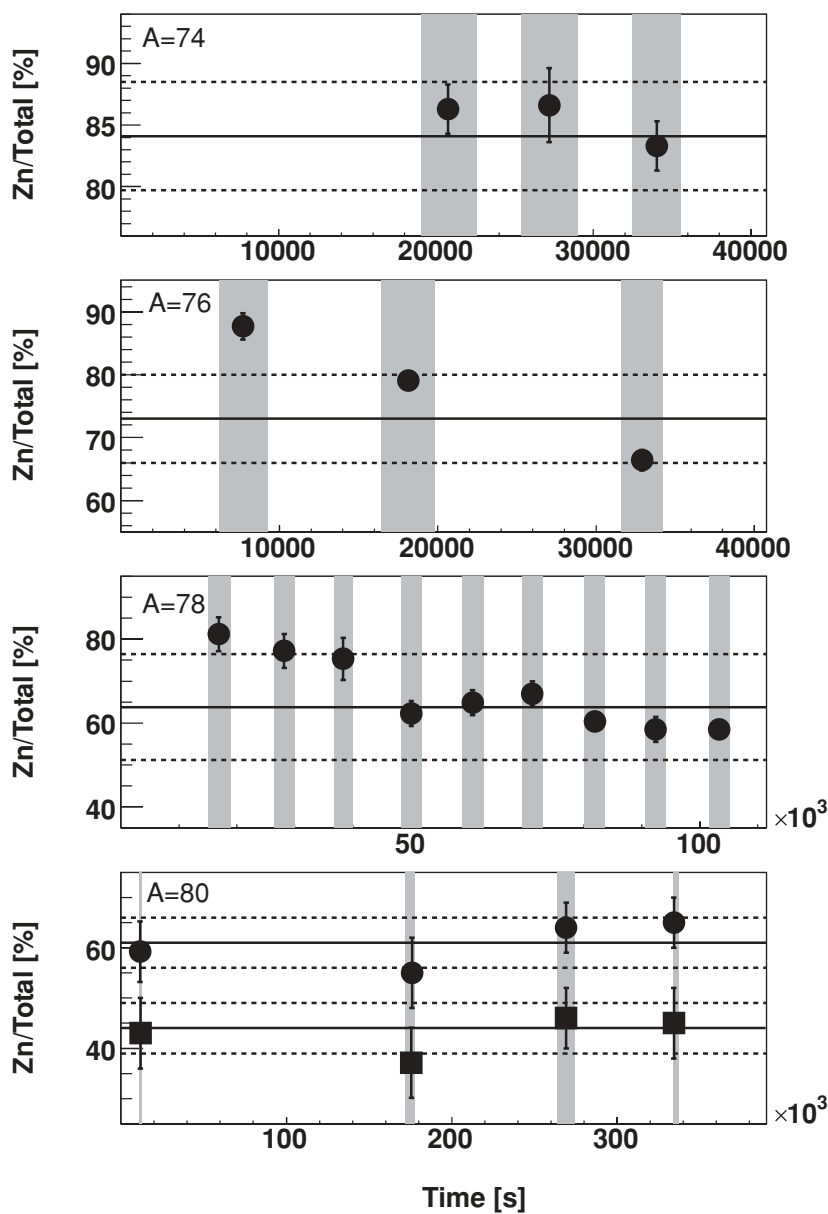

FIG. 7. The $\mathrm{Zn} /$ Total ratios during the $A=74-80$ experiments as a function of measurement time. The shaded time periods were measured in laser ON/OFF mode and the $\mathrm{Zn} /$ Total ratio is extracted from the difference in scattering rate in the DSSSD. The horizontal lines for $A=74,76,78$ correspond to the $\mathrm{Zn} /$ Total ratio, extracted from Coulomb excitation lines during the laser ON periods. The upper/lower horizontal lines for $A=80$ correspond to the average $\mathrm{Zn} /$ Total ratio during laser $\mathrm{ON} / \mathrm{OFF}$ measurements, in the time interval $150-650 / 0-2400 \mathrm{~ms}$ after the proton impact.

For the $A=78$ beam, the ratio $\frac{I_{\mathrm{Ga}}^{s}}{f_{\mathrm{Zn}} I^{l}}$, measured during the laser ON/OFF periods, was related to the observed $\mathrm{Ga}$ and target de-excitation $\gamma$-rays (the $281-\mathrm{keV} \gamma$ ray from ${ }^{78} \mathrm{Ga}$ and $434-\mathrm{keV} \gamma$ ray from ${ }^{108} \mathrm{Pd}$ ), because the statistics in the $730-\mathrm{keV}$ transition from ${ }^{78} \mathrm{Zn}$ is too low during the laser ON/OFF periods. This yields the following relation:

$$
\kappa_{2}=\frac{N_{\gamma, \mathrm{Ga}}}{N_{\gamma, T}^{\mathrm{tot}}} \times \frac{2+\frac{f_{\mathrm{Zn}} I}{I_{\mathrm{Ga}}^{s}} \times\left(\frac{\sigma_{T}^{\mathrm{Zn}}}{\sigma_{T}^{\mathrm{Ga}}}+\frac{f_{\mathrm{Ga}}}{f_{\mathrm{Zn}}}\right)}{2+\frac{f_{\mathrm{Zn}} I}{I_{\mathrm{Ga}}^{s}} \times \frac{f_{\mathrm{Ga}}}{f_{\mathrm{Zn}}}}
$$

and the ratio $\frac{I_{\mathrm{Ga}}}{f_{\mathrm{Zn}} I^{l}}$ during laser $\mathrm{ON}$ runs is thus given by

$$
\left(\frac{I_{\mathrm{Ga}}^{s}}{f_{\mathrm{Zn}} I^{l}}\right)^{\mathrm{ON}}=\frac{\frac{N_{\gamma, \mathrm{Ga}}}{N_{\gamma, T}^{\mathrm{ot}}} \times\left(\frac{\sigma_{T}^{\mathrm{Zn}}}{\sigma_{T}^{\mathrm{Ga}}}+\frac{f_{\mathrm{Ga}}}{f_{\mathrm{Zn}}}\right)-\kappa_{2} \times \frac{f_{\mathrm{Ga}}}{f_{\mathrm{Zn}}}}{\kappa_{2}-\frac{N_{\gamma, \mathrm{Ga}}}{N_{\gamma, T}^{\text {tot }}}} .
$$


The $\mathrm{Zn} /$ Total ratios for the $A=74,76,78$ beams, given by $\frac{f_{\mathrm{ZI}} I^{I}}{I^{\mathrm{I}}+I_{\mathrm{S}}^{s}}$, are plotted for each laser ON/OFF measurement in Fig. 7 and are summarized in Table II in the column "ON/OFF." The horizontal lines in Fig. 7 indicate this ratio during laser ON periods, determined with the methods described above. The dashed lines indicate the error bar on the ratio during the laser ON periods. These values are summarized in Table II in the column "ON." The $B\left(E 2,2_{1}^{+} \rightarrow 0_{1}^{+}\right)$values for ${ }^{74,76,78} \mathrm{Zn}$, given in Table II were determined using the $\mathrm{Zn} /$ Total ratio from the column "ON." The $B\left(E 2,4_{1}^{+} \rightarrow 2_{1}^{+}\right)$values for ${ }^{74,76} \mathrm{Zn}$ do not depend strongly on the $\mathrm{Zn} / \mathrm{Total}$ ratios and are given in Table II as well.

\section{2. ${ }^{80} \mathrm{Zn}$}

As discussed above, two isobaric contaminants were identified in the $A=80$ beam: ${ }^{80} \mathrm{Ga}$ and ${ }^{80} \mathrm{Rb}$. The normalized target yield, defined in Eq. (1), becomes:

$$
\begin{aligned}
N_{\gamma, T}^{\mathrm{Zn}}= & N_{T}^{\text {Total }} \\
& \times\left[1+\left(\frac{f_{\mathrm{Ga}}}{f_{\mathrm{Zn}}}+\frac{I_{\mathrm{Ga}}^{s}}{f_{\mathrm{Zn}} I^{l}}\right) \times \frac{\sigma_{T}^{G a}}{\sigma_{T}^{\mathrm{Zn}}}+\frac{I_{\mathrm{Rb}}^{s}}{f_{\mathrm{Zn}} I^{l}} \times \frac{\sigma_{T}^{\mathrm{Rb}}}{\sigma_{T}^{\mathrm{Zn}}}\right]^{-1} .
\end{aligned}
$$

The laser ON/OFF method was used as well and 6\% of the measuring time was dedicated to these laser ON/OFF measurements. These periods are indicated in Fig. 7 with the shaded areas. From the difference in scattering rate in the DSSSD during laser ON and laser OFF periods, the following relation can be established during laser ON/OFF measurements:

$$
\frac{N^{\mathrm{OFF}}}{N^{\mathrm{ON}}-N^{\mathrm{OFF}}}=R=\frac{I_{\mathrm{Ga}}^{s} \sigma_{R}^{\mathrm{Ga}}+I_{\mathrm{Rb}}^{s} \sigma_{R}^{\mathrm{Rb}}}{f_{\mathrm{Zn}} \times I^{l} \sigma_{R}^{\mathrm{Zn}}+f_{\mathrm{Ga}} \times I^{l} \sigma_{R}^{\mathrm{Ga}}},
$$

where $\sigma_{R}^{x}$ is the elastic Rutherford cross section for element $x$. Combining Eq. (8) and Eq. (9), the normalized target yield is given by:

$$
\begin{aligned}
& N_{\gamma, T}^{\mathrm{Zn}}=N_{\gamma, T}^{\mathrm{Tota}} \\
& \times\left\{1+\frac{\sigma_{T}^{\mathrm{Ga}}}{\sigma_{T}^{\mathrm{Zn}}} \times\left[\frac{f_{\mathrm{Ga}}}{f_{\mathrm{Zn}}}+\frac{1+\frac{I_{\mathrm{Rb}}^{s}}{I_{\mathrm{Ga}}^{s}} \frac{\sigma_{T}^{\mathrm{Rb}}}{\sigma_{T}^{\mathrm{Ga}}}}{1+\frac{I_{\mathrm{Rb}}^{s}}{I_{\mathrm{Ga}}^{s}} \frac{\sigma_{R}^{\mathrm{Rb}}}{\sigma_{R}^{\mathrm{Ga}}}} \times R \times\left(\frac{\sigma_{R}^{\mathrm{Zn}}}{\sigma_{R}^{\mathrm{Ga}}}+\frac{f_{\mathrm{Ga}}}{f_{\mathrm{Zn}}}\right)\right]\right\}^{-1} .
\end{aligned}
$$

The ratio of surface ionized ${ }^{80} \mathrm{Ga}$ and ${ }^{80} \mathrm{Rb}\left(I_{\mathrm{Rb}}^{s} / I_{\mathrm{Ga}}^{s}\right)$ was extracted from the intensity of their most intense $\beta$-decay lines: $659 \mathrm{keV}\left({ }^{80} \mathrm{Ga}, I_{\gamma}=78(2) \%[20]\right)$ and $616 \mathrm{keV}\left({ }^{80} \mathrm{Rb}\right.$, $I_{\gamma}=25(3) \%$ [20]), observed in the MINIBALL array. The amount of $659-\mathrm{keV} \gamma$ rays from the $\beta$-decay of surface ionized gallium was extracted from a comparison of the singles $\gamma$-ray spectrum during the last $10 \mathrm{~s}$ of the laser OFF period during the laser ON/OFF experiments (periodicity was $21.6 \mathrm{~s}$ ). During this time period, the zinc and gallium activity from the laser ON period has diminished to $<1 \%$ [the half-life of ${ }^{80} \mathrm{Ga}$ is $1.676(14) \mathrm{s}]$.

Contrary to the normalization methods used for ${ }^{74,76,78} \mathrm{Zn}$, no correlation could be made between the parameter " $R$ " and prompt de-excitation $\gamma$ rays from the main contaminants

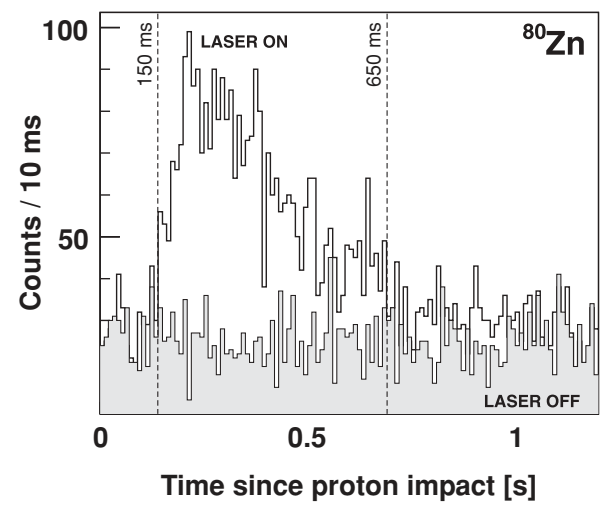

FIG. 8. Detected number of particles in the DSSSD as a function of the arrival time in the DSSSD after the proton pulse impact on the primary target. Two time periods were selected to integrate the Coulomb excitation spectra, one from 150 to $650 \mathrm{~ms}$ and a second from 0 from $2400 \mathrm{~ms}$. The $\mathrm{Zn} /$ Total ratios over these two time periods are given in Table II.

during laser ON/OFF periods, due to the lacking statistics in the Coulomb excitation spectra during laser ON/OFF measurements. Therefore, the value " $R$," measured during laser ON/OFF periods was straightforwardly applied to the laser ON data. Two time periods were selected in both laser $\mathrm{ON}$ and laser ON/OFF runs. One period spans 150 to $650 \mathrm{~ms}$ after the proton impact. During this period, the bulk part of zinc isotopes has diffused out of the primary target (see Fig. 8). The second period spans from 0 to $2400 \mathrm{~ms}$ after the proton impact and corresponds to the full measuring period, because the maximum separation between proton pulses was $2.4 \mathrm{~s}$. The average $\mathrm{Zn} /$ Total ratios during the laser ON/OFF periods (now given by $\frac{f_{\mathrm{Z} I} I^{l}}{I^{l}+I_{\mathrm{Ga}}^{s}+I_{\mathrm{Rb}}^{s}}$ ) and the resulting $B(E 2 \downarrow)$ for both time periods, are given in Table II. The circles(squares) in Fig. $7(A=80)$ correspond to $\mathrm{Zn} /$ Total ratios during individual laser ON/OFF measurements in the 150-650(0-2400) ms time period after the proton impact, respectively. The horizontal lines correspond to the average $\mathrm{Zn} /$ Total ratios during the laser ON/OFF measurements.

During the $A=80$ experiment, an extended amount of time was spent with the lasers off. The sum of all laser OFF spectra is shown in Fig. 6(c). A large amount of target excitation is observed at $434 \mathrm{keV}$, together with three prompt $\gamma$ rays at 175,201 , and $376 \mathrm{keV}$, which are $\gamma$ rays de-exciting known excited levels in ${ }^{80} \mathrm{Rb}$. From the laser OFF data, a relation can be established between the amount of target excitation induced by surface ionized rubidium and gallium $\left(N_{\gamma}^{\mathrm{Rb}+\mathrm{Ga}}\right)$, the observed amount of $175-\mathrm{keV} \gamma$ rays coming from ${ }^{80} \mathrm{Rb}$ $\left(N_{\gamma, \mathrm{Rb}}\right)$ and the $I_{\mathrm{Ga}}^{s} / I_{\mathrm{Rb}}^{s}$ ratio:

$$
N_{\gamma, T}^{\mathrm{Rb}+\mathrm{Ga}}=\kappa_{3} \times N_{\gamma, \mathrm{Rb}} \times\left(1+\frac{I_{\mathrm{Ga}}^{s}}{I_{\mathrm{Rb}}^{s}} \times \frac{\sigma_{T}^{\mathrm{Ga}}}{\sigma_{T}^{\mathrm{Rb}}}\right) .
$$

The obtained parameter $\kappa_{3}$ can be used during the laser ON periods to deduce the amount of target excitation induced by surface-ionized rubidium and gallium from the number of counts in the $175-\mathrm{keV} \gamma$ ray. Thus, the number of target de-excitation counts induced by ${ }^{80} \mathrm{Zn}$ during laser $\mathrm{ON}$ periods 
is given by:

$$
N_{\gamma, T}^{\mathrm{Zn}}=\left(N_{\gamma, T}^{\mathrm{tot}}-N_{\gamma, T}^{\mathrm{Rb}+\mathrm{Ga}}\right) \times\left(1+\frac{f_{\mathrm{Ga}}}{f_{\mathrm{Zn}}} \times \frac{\sigma_{T}^{\mathrm{Ga}}}{\sigma_{T}^{\mathrm{Zn}}}\right)^{-1} .
$$

The $175-\mathrm{keV} \gamma$ ray from ${ }^{80} \mathrm{Rb}$ was integrated over the same two time periods as defined above (150-650 and 0-2400 ms after the proton impact). The $\mathrm{Zn} /$ Total ratios during the laser ON period deduced in this manner are given in Table II in the column "ON" and these are consistent with the $\mathrm{Zn}$ /Total ratios measured during the laser ON/OFF periods. The horizontal lines in Fig. 7 correspond to the most precise $\mathrm{Zn} /$ Total ratios, which are the ratios deduced from the laser ON/OFF method. The resulting $B\left(E 2,2_{1}^{+} \rightarrow 0_{1}^{+}\right)$values for each normalization method of the $A=80$ experiment are summarized in Table II. We adopt the value with the lowest relative error as our final result: $B\left(E 2,2_{1}^{+} \rightarrow 0_{1}^{+}\right)=144(29) e^{2} \mathrm{fm}^{4}$.

\section{Quadrupole moment and higher-lying states}

The resulting $B\left(E 2,2_{1}^{+} \rightarrow 0_{1}^{+}\right)$values depend strongly on the sign and magnitude of the diagonal matrix element $M_{2,2}=\left\langle 2_{1}^{+}\|E 2\| 2_{1}^{+}\right\rangle$, related to the spectroscopic quadrupole moment: $Q_{2_{1}^{+}}=0.7579 \times M_{2,2}$. In Fig. 9, the resulting lifetime of the $2_{1}^{+}$state $\left[\tau_{2_{1}^{+}}(p s)=40.81 \times 10^{13} \times\right.$ $\left.E_{2_{1}^{+}, \mathrm{keV}}^{-5} \times B\left(E 2,0_{1}^{+} \rightarrow 2_{1}^{+}\right)_{e^{2} \mathrm{~b}^{2}}^{-1}\right]$ is plotted as a function of the quadrupole moment. These results were obtained by fixing the diagonal matrix element to values between -0.5 and $0.5 \mathrm{eb}$ in GOSIA2. The results at $Q_{2_{1}^{+}}=0.0 \mathrm{eb}$ correspond to the $B(E 2 \downarrow)$ values in Table II [for $A=80$, the $B(E 2 \downarrow)$ with the smallest error bar]. Additional information on the sign and magnitude of the quadrupole moment could be obtained

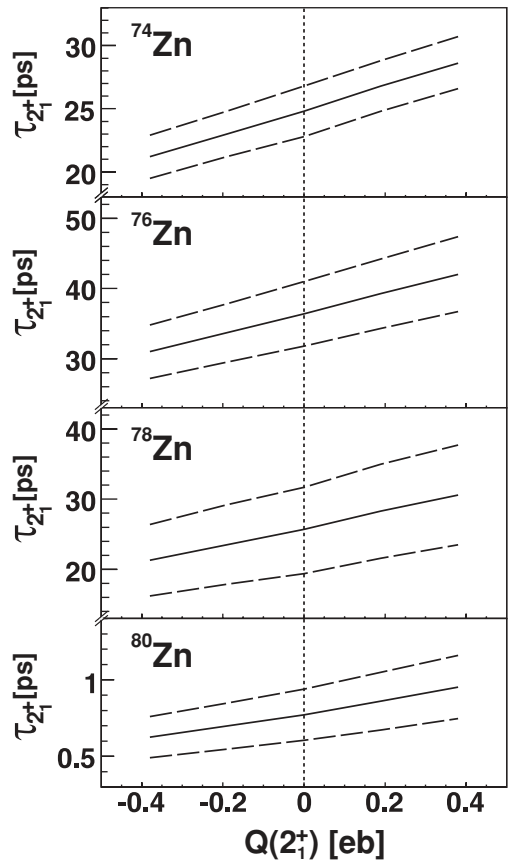

FIG. 9. Dependence of the experimental $\tau_{2_{1}^{+}}$values on the fixed sign and magnitude of quadrupole moment $\left(Q_{2_{1}^{+}}\right)$in GOSIA2. The dashed lines indicate the error bars associated with the lifetimes. by combining lifetime measurements of the $2_{1}^{+}$state and the data in Fig. 9.

Higher-lying states were included in the calculation of the $\gamma$-ray yield. In the case of ${ }^{74} \mathrm{Zn}$, the $4_{1}^{+}$state at $1419 \mathrm{keV}$ and the $2_{2}^{+}$state at $1670 \mathrm{keV}$ were included and for ${ }^{76} \mathrm{Zn}$ only the $4_{1}^{+}$ state at $1296 \mathrm{keV}$ was included. Experimentally, the $4_{1}^{+} \rightarrow 2_{1}^{+}$ transitions were observed in the ${ }^{74} \mathrm{Zn}$ and ${ }^{76} \mathrm{Zn}$ Dopplercorrected spectra (see Fig. 3 and Fig. 4). These transitions have a relative intensity of $1.4(2) \%(A=74)$ and $1.4(5) \%(A=$ 76) of the respective $2_{1}^{+} \rightarrow 0_{1}^{+}$transition. The $\left\langle 2_{1}^{+} \| E 2|| 4_{1}^{+}\right\rangle$ matrix elements could be determined from these experimental intensities, resulting in the $B\left(E 2,4_{1}^{+} \rightarrow 2_{1}^{+}\right)$values in Table II. The influence of this $\left\langle 2_{1}^{+}\|E 2\| 4_{1}^{+}\right\rangle$matrix element on the calculated $2_{1}^{+} \rightarrow 0_{1}^{+} \gamma$-ray yields remains below $0.5 \%$ in the limit of a vibrational $B\left(E 2,2_{1}^{+} \rightarrow 4_{1}^{+}\right)$value for both masses. The determined $B\left(E 2,4_{1}^{+} \rightarrow 2_{1}^{+}\right)$changes by $\pm 6 \%$ for a quadrupole moment of $\pm 0.38 \mathrm{eb}$ for both masses. No counts are observed around the $2_{2}^{+} \rightarrow 2_{1}^{+}$transition at $1064 \mathrm{keV}$ in the $A=74$ Doppler corrected spectrum. From the nonobservation of this transition, an upper limit can be put on the $B\left(E 2,2_{2}^{+} \rightarrow 2_{1}^{+}\right)$value of $520 e^{2} \mathrm{fm}^{4}$, assuming $0.1 \%$ of the $2_{1}^{+} \rightarrow 0_{1}^{+}$transition yield is below the observation limit. For ${ }^{78} \mathrm{Zn}$, the $\left(4_{1}^{+}\right)$state at $1621 \mathrm{keV}$ was included in the calculation and the influence of a vibrational $B\left(E 2,4_{1}^{+} \rightarrow 2_{1}^{+}\right)$value on the $B\left(E 2,2_{1}^{+} \rightarrow 0_{1}^{+}\right)$is in this case as well below $0.5 \%$. For ${ }^{80} \mathrm{Zn}$ no higher-lying states were included in the calculation.

\section{DISCUSSION}

In Fig. 10 the $B\left(E 2,2_{1}^{+} \rightarrow 0_{1}^{+}\right)$reduced transition probabilities (denoted by $B(E 2 \downarrow)$ in the remaining discussion) and $E\left(2_{1}^{+}\right)$systematics is shown for isotopes around $Z=28$ : iron $(Z=26)$, nickel $(Z=28)$, zinc $(Z=30)$, germanium $(Z=32)$, and selenium $(Z=34)$ as a function of neutron number $N$. Inspecting the $E\left(2_{1}^{+}\right)$behavior with the neutrons filling the $2 p_{3 / 2}, 1 f_{5 / 2}, 2 p_{1 / 2}$, and $1 g_{9 / 2}$ orbitals from $N=28$

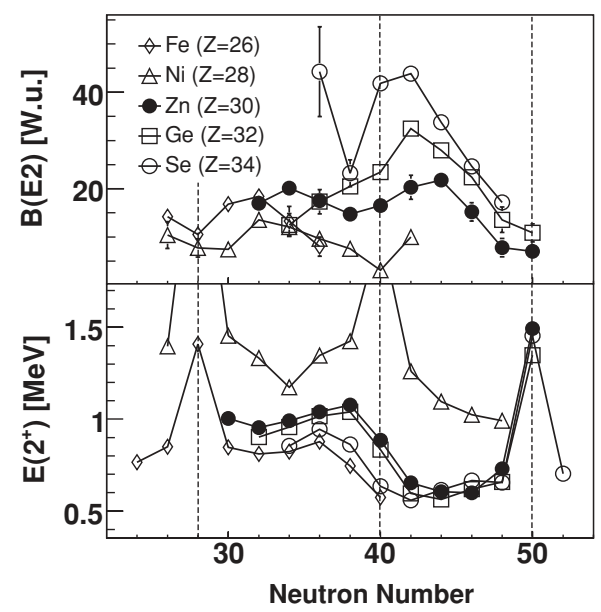

FIG. 10. Systematics of the $B\left(E 2,2_{1}^{+} \rightarrow 0_{1}^{+}\right)$and $E\left(2_{1}^{+}\right)$values for iron $(Z=26)$, nickel $(Z=28)$, zinc $(Z=30)$, germanium $(Z=32)$, and selenium $(Z=34)$ isotopes. Data are taken from Refs. [13,20,38]. 
toward $N=50$, there are some conspicuous observations to be made. Only in the case of $\mathrm{Ni}(Z=28)$ does the proton shell closure allow for a stabilization of both the $N=28$ shell and $N=40$ subshell closures. Removing just two protons (the iron nuclei), or adding proton pairs beyond $Z=28$ (zinc, germanium and selenium isotopes), the peak in the $E\left(2_{1}^{+}\right)$value at $N=40$ quickly disappears indicating the importance of proton-neutron correlations that seem to dominate over the normal filling of the neutron single-particle filling as implied by the monopole term. Interesting to note also is the clear difference in the energy scale for the $2_{1}^{+}$first excited state comparing the region $28 \leqslant N \leqslant 38$ (with a typical energy of $0.8-1.0 \mathrm{MeV}$ ) and the $40 \leqslant N \leqslant 50$ region (with a typical energy of $0.6 \mathrm{MeV}$ ), which are otherwise very similar irrespective of a proton hole (iron) or proton particle (zinc, germanium, selenium) character. This difference indicates an increased collectivity in the heavier nuclei and is clearly correlated with the filling of the neutron $1 g_{9 / 2}$ orbital.

The sudden decrease of the $2_{1}^{+}$states in zinc, germanium, and selenium at $N=40$, accompanied with an increase of the $B(E 2 \downarrow)$ strength, indicates an increase in collectivity around $N=40$. In germanium isotopes, this enhanced collectivity leads to a deformed ground-state configuration in ${ }^{72} \mathrm{Ge}$, which is found to be a $\gamma$-soft nucleus [39] and shape coexistence appears in both germanium and selenium isotopes with $N>$ 40. The increased collectivity in zinc isotopes with $N>40$ continues up to $N=44$, as reflected from the measured $B(E 2 \downarrow)$ value in ${ }^{74} \mathrm{Zn}$ in this work and in Ref. [13], which are in agreement with each other. The enhanced collectivity in ${ }^{70-74} \mathrm{Zn}$ can be understood from a weak $N=40$ shell gap, a maximum in neutron pairing correlations and the two protons outside the $Z=28$ shell gap [40,41]. Across midshell, at $N=46$, the collectivity suddenly drops. From the measured $B(E 2 \downarrow)$ values, deformation parameters $\beta_{2}$ can be deduced using the expression for vibrational motion [36]

$$
B\left(E 2 ; 0_{1}^{+} \rightarrow 2_{1}^{+}\right)=\left(\frac{3}{4 \pi} \mathrm{Ze} R_{0}^{2}\right)^{2} \beta_{2}^{2},
$$

resulting in values of $0.25(1), 0.21(1), 0.15(2)$, and $0.14(2)$ for the $A=74,76,78$, and 80 zinc isotopes. If we consider the extracted value of $\beta_{2}$ as a qualitative measure of deformation, one can consider the structure of the Nilsson single-particle spectrum near the value of $\beta_{2}=0.25$. It turns out that the lowest-lying three Nilsson states originating from the spherical $1 g_{9 / 2}$ orbital are all strongly downsloping for both prolate and oblate deformation, which would make deformation energetically less favorable in ${ }^{78,80} \mathrm{Zn}$.

A few interesting quantities such as the energy ratio $R_{4 / 2} \equiv E_{x}\left(4_{1}^{+}\right) / E_{x}\left(2_{1}^{+}\right)$and the $B(E 2 \downarrow)$ ratio $B\left(E 2,4_{1}^{+} \rightarrow\right.$ $\left.2_{1}^{+}\right) / B\left(E 2,2_{1}^{+} \rightarrow 0_{1}^{+}\right)$give a qualitative idea on the collective properties characterizing the low-energy nuclear structure in atomic nuclei. In Fig. 11, we present those ratios for the zinc isotopic chain. The $R_{4 / 2}$ ratio in the range 2 to 2.5 are typical values for nuclei that exhibit an apparent vibrational character. It turns out that for all zinc nuclei, the $R_{4 / 2}$ ratio is within that range [31]. There is a most interesting modulation with the lower values correlating with the neutron number $N=40$ and most probably with $N=28$ and $N=28$ that hint at a more

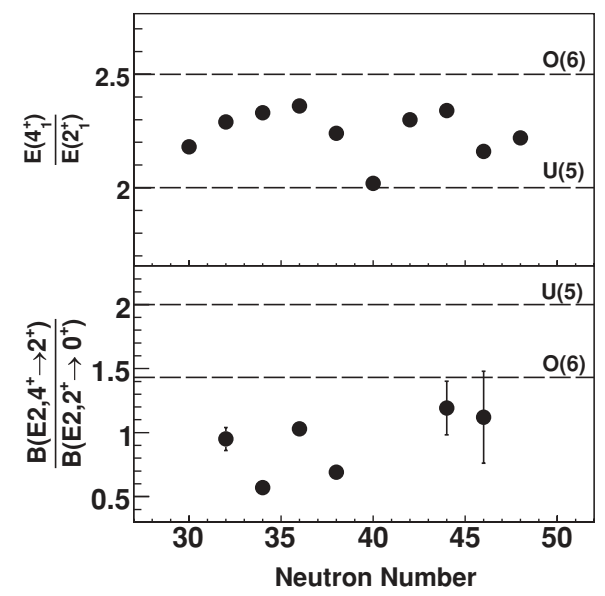

FIG. 11. The $R_{4 / 2}$ and $B\left(E 2,4_{1}^{+} \rightarrow 2_{1}^{+}\right) / B\left(E 2,2_{1}^{+} \rightarrow 0_{1}^{+}\right)$ratios for zinc isotopes. Data are taken from Refs. [13,40,44-47].

rigid structure near these values, in particular for $N=40$, showing a remaining effect of the $N=40$ subshell closure. The available $B(E 2 \downarrow)$ ratios in ${ }^{62,64,66,68} \mathrm{Zn}$ and the new values for the radioactive ${ }^{74,76} \mathrm{Zn}$, which are of the order of 1 , or even less, do not support such a simple vibrational picture in which, for pure harmonic vibrations, this ratio is 2 . This vibrational picture is also equivalent with the large boson number limit of the IBM-1 U(5) symmetry [42]. In the same model, there exists an interesting symmetry, the $\mathrm{O}(6)$ symmetry, which can be characterized by a $R_{4 / 2}$ ratio of 2.5 and a $B(E 2 \downarrow)$ ratio of $10 / 7$ (in the large boson number limit). This $\mathrm{O}(6)$ symmetry is equivalent to $\gamma$-soft nuclei when using a geometrical picture and the above ratios become exactly 2.5 and 10/7, respectively, in the extreme $\gamma$-independent model of Jean-Wilets [43]. The characteristic $R_{4 / 2}$ and $B(E 2 \downarrow)$ ratios are shown in Figs. 11(a) and 11(b) for those particular limits of the $U(5)$ and $O(6)$ limit such that no boson (neutron number) dependence remains (which is not the case in more realistic studies). These lines also correspond exactly with the geometrical harmonic vibrational and $\gamma$-independent values. From the limited experimental data in Fig. 11, one can conclude that the low-energy structure of zinc isotopes is indicative for $\gamma$ softness or resembles more closely the $\mathrm{O}(6)$ symmetry. Evidence for this was found as well from a multiple Coulomb excitation study of ${ }^{68} \mathrm{Zn}$, where transition strengths connecting states up to $2.5 \mathrm{MeV}$ give relatively good agreement with the $\mathrm{O}(6)$ symmetry [44]. Inspecting the detailed level structure in the zinc isotopes, up to an energy of $\approx 3 \mathrm{MeV}$, these energy spectra exhibit far more complex structure that goes beyond simple collective models.

In Fig. 12, the experimental $B(E 2 \downarrow)$ values for the zinc isotopic chain are compared to $B(E 2 \downarrow)$ values, calculated with the revised Grodzins rule (dashed line) from Ref. [36]. Because few valence nucleons are present in the zinc isotopes and a strong stabilizing effect is expected from the $N=50$ and $Z=28$ shell closures it is expected that this rule has limited predictive power in this region of the nuclear chart. A reasonable agreements is found for the whole isotopic chain when the calculated values are scaled with a factor 0.92 (as discussed in Ref. [36], a simple renormalization brings the predictions in good agreement). Of course, the remarkable 


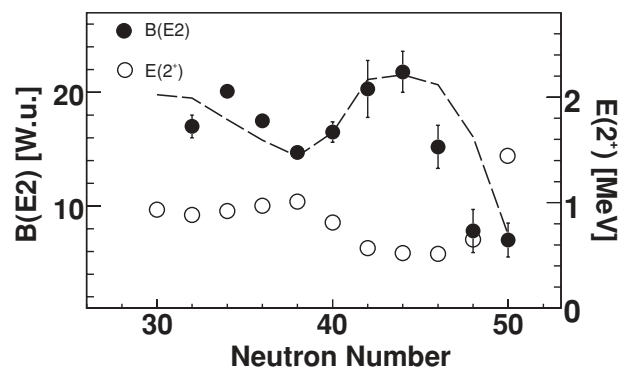

FIG. 12. Experimental $E\left(2_{1}^{+}\right)$(open circles) and $B(E 2 \downarrow)$ values (filled circles) for zinc isotopes and $B(E 2 \downarrow)$ values predicted by the Grodzins rule from Ref. [36] (dashed line), using the experimental $E\left(2_{1}^{+}\right)$.

agreement for zinc isotopes is no guarantee that this rule applies to other isotopes with extreme $N / Z$ ratios in this or other regions of the nuclear chart.

Recently, the $B(E 2 \downarrow)$ values for radioactive ${ }^{78,80,82} \mathrm{Ge}$ were measured by low-energy Coulomb excitation [38]. A dramatic drop in $B(E 2 \downarrow)$ strength was observed from $N=$ 42 down to $N=50$ in germanium isotopes, similar to the $B(E 2 \downarrow)$ trend in selenium isotopes $\left({ }^{74-84} \mathrm{Se}\right)$ and in the current results for zinc isotopes. In Ref. [38], it was concluded that $N=50$ remains a good shell closure for the $N / Z$ ratio $1.56\left({ }^{82} \mathrm{Ge}\right)$, based on the excellent agreement between the experimental results and shell-model calculations, using a preliminary version of the JJ4B interaction (see below) $[38,48]$, which does not include neutron excitations across $N=50$.

To reach a microscopic understanding of the observed trends, large-scale shell-model studies have to be carried out for the present nuclei. In Fig. 13, the $(B E 2 \downarrow)$ and $E\left(2_{1}^{+}\right)$ systematics for the zinc isotopes are compared with the results of two sets of such calculations, both based on the renormalized matrix starting from the same Bonn potential [49]. In the first calculation (labeled SMI), the realistic effective nucleon-nucleon interaction based on $G$-matrix theory, constructed by M. Hjorth-Jensen et al. [49], including the monopole modification for the model space, as determined

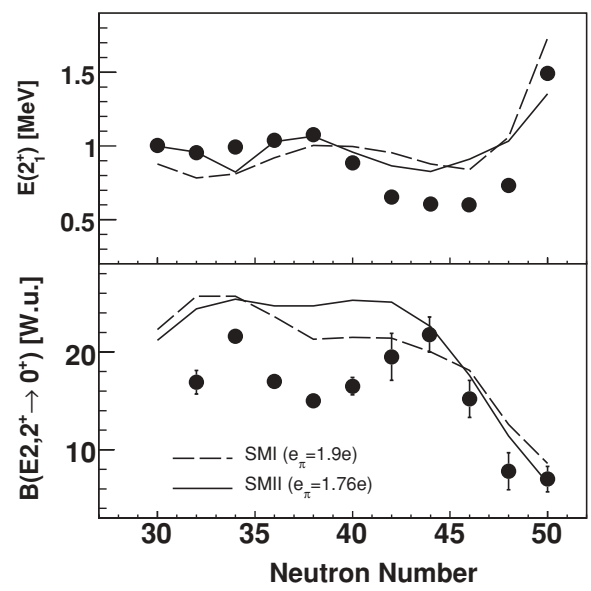

FIG. 13. Experimental $E\left(2_{1}^{+}\right)$and $B\left(E 2,2_{1}^{+} \rightarrow 0_{1}^{+}\right)$values for zinc isotopes, compared to shell-model calculations. See text for details on these calculations. by Nowacki [50,51], was used. The actual diagonalization was carried out using the shell-model code NATHAN [1,52]. The model space consists of the $2 p_{3 / 2}, 1 f_{5 / 2}, 2 p_{1 / 2}$, and $1 g_{9 / 2}$ orbitals for both protons and neutrons moving outside of an inert ${ }^{56} \mathrm{Ni}$ core. The input single particle energies were $\epsilon_{1 f_{5 / 2}}-\epsilon_{2 p_{3 / 2}}=0.77 \mathrm{MeV}, \epsilon_{2 p_{1 / 2}}-\epsilon_{2 p_{3 / 2}}=1.11 \mathrm{MeV}$ and $\epsilon_{1 g_{9 / 2}}-\epsilon_{2 p_{3 / 2}}=3.00 \mathrm{MeV}$ for proton and neutron orbitals. Quite large $\pi$ and $v$ effective charges of $1.9 e / 0.9 e$ were used to compensate for large ${ }^{56} \mathrm{Ni}$ core polarization that are clearly present. Shell-model and Monte Carlo SM calculations have shown that the doubly closed shell probability of ${ }^{56} \mathrm{Ni}$ is only $\sim 65 \%$ [53] and $\sim 50 \%$ [54]. The second calculation (labeled SMII) was carried out using the JJ4B effective interaction [55]. This interaction was constructed, also starting from the renormalized $G$-matrix interaction [based on the Bonn-C NN potential (JJ4APN)], but adjustments to linear combinations of the matrix elements have been made to reproduce the experimental data for exotic $\mathrm{Ni}, \mathrm{Cu}, \mathrm{Zn}, \mathrm{Ge}$, and $N=50$ isotones in the vicinity of ${ }^{78} \mathrm{Ni}$. Proton and neutron polarization charges of $1.76 e$ and $0.97 e$, respectively, were used reproducing known $B\left(E 2,8_{1}^{+} \rightarrow 6_{1}^{+}\right)$values in ${ }^{92} \operatorname{Mo}\left(e_{\pi}\right)$ and ${ }^{70} \mathrm{Ni}\left(e_{\nu}\right)$. Harmonic-oscillator radial wave functions were used with $\hbar \omega=45 A^{-\frac{1}{3}}-25 \mathrm{~A}^{-\frac{3}{2}}$. The JJ4B interaction is the first "empirical interaction" in the $p f_{5 / 2} g$ shell that has become available and that takes into account experimental information from rarely produced radioactive isotopes up to the year 2004.

Both shell-model calculations SMI and SMII reproduce the experimental $B(E 2 \downarrow)$ trend for the zinc isotopic chain rather well. The downward $B(E 2 \downarrow)$ trend for the neutron-rich zinc isotopes with $N \geqslant 44$ is nicely reproduced. The discrepancy around $N=40$, where both SMI and SMII overestimate the $B(E 2 \downarrow)$ strength to the first excited $2_{1}^{+}$state, can be understood from the position of the $v 1 g_{9 / 2}$ orbit and its influence on the $0_{2}^{+}$energy state [56]. Particularly illustrative for this effect is the fact that the summed experimental $B(E 2 \downarrow)$ strengths $B\left(E 2,2_{1}^{+} \rightarrow 0_{1}^{+}\right)+\mathrm{B}\left(E 2,2_{1}^{+} \rightarrow 0_{2}^{+}\right)=411(22) e^{2} \mathrm{fm}^{4}$ for ${ }^{70} \mathrm{Zn}$, which compares well with the calculated $B\left(E 2,2_{1}^{+} \rightarrow\right.$ $0_{1}^{+}$) strength of $434 e^{2} \mathrm{fm}^{4}$ (see the SMII results). An extensive shell-model study of the influence of the neutron $1 g_{9 / 2}$ orbital on $B(E 2 \downarrow)$ strengths in germanium isotopes can be found in Ref. [56].

In Fig. 14, the $B(E 2 \downarrow)$ and $E\left(2_{1}^{+}\right)$systematics is shown for the $N=50$ isotones. Historically, $\mathrm{Ji}$ and Wildenthal developed the first "empirical residual interaction" for the $N=50$ isotones. This interaction was fitted to experimental data available up to the year 1987. The calculated excitation energies $E\left(2_{1}^{+}\right)$(taken from Ref. [57]) are shown in Fig. 14 (the dotted line). These early calculations reproduce the available $E\left(2_{1}^{+}\right)$systematics known up to $1987(Z=32-46)$ rather well. Remarkably, the energy of the $2_{1}^{+}$state in ${ }^{80} \mathrm{Zn}$ is calculated $60 \mathrm{keV}$ below the $2_{1}^{+}$state in ${ }^{82} \mathrm{Ge}$, whereas an increase would be expected for a strong $Z=28$ shell closure. The full line in Fig. 14 shows the calculations resulting from SMII (which makes use of the new empirical interaction, called JJ4B, which was also fitted to experimental data in the $N=50$ isotones up to the year 2004, see above). This new interaction accounts very well for the $E\left(2_{1}^{+}\right)$excitation energy in isotopes with $Z<40$ but underestimates the experimental 


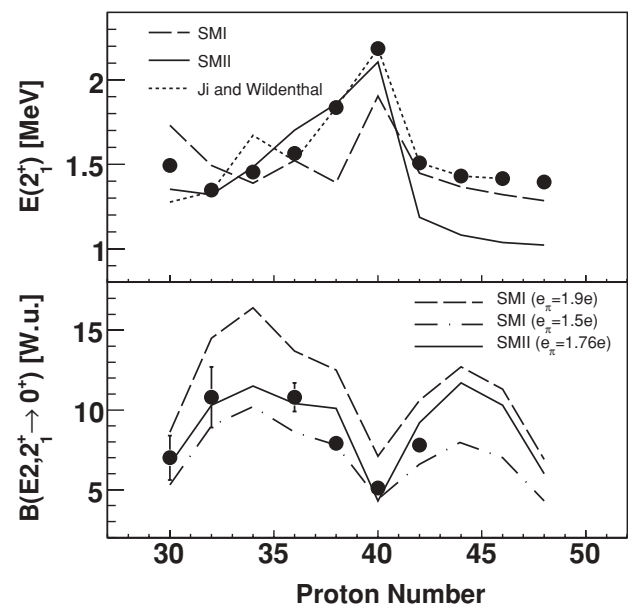

FIG. 14. $E\left(2_{1}^{+}\right)$and $B(E 2 \downarrow)$ systematics of $N=50$ isotones. Values from this work are indicated with squares. The experimental $2_{1}^{+}$energies are compared to calculations taken from Ref. [57], SMI and SMII calculations. The experimental $B(E 2 \downarrow)$ values are compared to SMI calculations with $e_{\pi}=1.9 e$ and $1.5 e$ and SMII calculations with $e_{\pi}=1.76 e$. (See text for details.)

energies for the region $Z>40$ isotones. The calculated $2_{1}^{+}$ energy in ${ }^{80} \mathrm{Zn}$ is only $77 \mathrm{keV}$ higher than in the early Ji-Wildenthal interaction; however, a higher energy is calculated compared to ${ }^{82} \mathrm{Ge}$. These two empirical residual interactions illustrate the extreme importance of new experimental data on exotic nuclei to develop a more general interaction. The SMI calculation (dashed line), however, performs very well for $N=50$ isotones with $Z>40$ but deviates for the lighter isotopes with $Z<40$, as compared to the SMII calculations.

The calculated $B(E 2 \downarrow)$ values within SMI are shown in Fig. 14 using both $e_{\pi}=1.9 e$ (the same effective proton charge as for zinc isotopes, dashed line) and a slightly lower value of $e_{\pi}=1.5 e$ (the standard effective proton charge, dash-dotted line). In the present model space (having a closed shell at $N=50$ ), no valence neutrons are available, so the $0_{1}^{+}$and $2_{1}^{+}$ states are pure proton states. The effective proton charge thus acts as a mere scaling factor in the calculation of the $B(E 2 \downarrow)$ value $\left[B(E 2 \downarrow) \sim e_{\pi}^{2} M_{\pi}^{2}\right]$. Using the same proton effective charge as for zinc isotopes $(1.9 e)$, the $B(E 2 \downarrow)$ values are generally overestimated. Making use of the standard effective proton charge of $1.5 e$, all values for isotopes with $Z<40$ are only slightly underestimated. The SMII calculation predicts the $B(E 2 \downarrow)$ strengths for $N=50$ isotones with $Z<40$ accurately using a proton effective charge of $1.76 e$, which was determined from fitting to the experimental $B\left(E 2,8_{1}^{+} \rightarrow 6_{1}^{+}\right)$ value in ${ }^{92} \mathrm{Mo}$. The empirical residual interaction SMII seems to include the effects of the polarization of the underlying core, ${ }^{56} \mathrm{Ni}$, rather well. It seems like the JJ4B interaction is able to reproduce rather well the recently obtained low-energy level structure properties of the $N=50$ isotope ${ }^{81} \mathrm{Ga}$ [58], illustrating the applicability of this interaction in the region near to ${ }^{78} \mathrm{Ni}$.

Based on the calculated $B(E 2 \downarrow)$ values for the $N=50$ isotones, no indication is found for a strong reduction of the $N=50$ shell gap at $Z=30$. The persistence of the $N=50$ shell gap is reported as well in Ref. [59], where high spin states in ${ }^{82} \mathrm{Ge}$ up to $4 \mathrm{MeV}$ are reproduced with shell-model calculations excluding neutron excitation across $N=50$ and in recent mass measurements [60].

\section{CONCLUSIONS}

The normalization of Coulomb excitation experiments with postaccelerated beams at ISOL facilities is hampered if considerable isobaric contamination of the radioactive ion beam occurs. In this work it has been shown how the selective laser ionization, available at REX-ISOLDE, can be used to deal with the isobaric beam contamination and how a reliable normalization can be achieved.

The Coulomb excitation experiments with postaccelerated radioactive ion beams of ${ }^{74,76,78,80} \mathrm{Zn}$ have resulted in the measurement of $B\left(E 2,2_{1}^{+} \rightarrow 0_{1}^{+}\right)$values in ${ }^{74-80} \mathrm{Zn}$, $B\left(E 2,4_{1}^{+} \rightarrow 2_{1}^{+}\right)$values in ${ }^{74,76} \mathrm{Zn}$, and the establishment of the $2_{1}^{+}$states in ${ }^{78,80} \mathrm{Zn}$. The available $R_{4 / 2}$ and $B(E 2 \downarrow)$ ratios for stable and radioactive zinc isotopes were compared to standard collective model predictions. The present data show a tendency toward characteristic behavior for the $\mathrm{O}(6)$ symmetry within the IBM model, or, equivalently, to $\gamma$ - unstable nuclei. The $B(E 2 \downarrow)$ and $E\left(2_{1}^{+}\right)$systematics for zinc isotopes and $N=50$ isotones was compared with large-scale shell-model calculations (labeled SMI and SMII). The new results for the zinc isotopes are accounted for reasonably well by the two calculations, provided a large proton effective charge of $1.9 e$ is used in SMI. The new empirical residual interaction JJ4B (used with the SMII calculations) performs overall very well in the calculation of the $B(E 2 \downarrow)$ and $E\left(2_{1}^{+}\right)$in the $N=50$ isotonic chain compared to the SMI calculations. This may be due to the fact that the new JJ4B has been constructed with the constraint of new experimental data in the most neutron-rich nuclei in this mass region. No direct evidence is found for an enhanced $Z=28$ core polarization, but the larger proton effective charge needed in the SMI calculations to describe $N=50$ isotones with $Z<40$ indicate a larger proton core polarization for these isotopes. No evidence is found for breaking of the $N=50$ shell gap.

\section{ACKNOWLEDGMENTS}

This work was supported by the European Union Sixth Framework through RII3-EURONS (Contract No. 506065), the German BMBF Grant 06KY205I, the BriX-IAP Research Program No. P06/23, and FWO-Vlaanderen (Belgium).
[1] E. Caurier et al., Rev. Mod. Phys. 77, 427 (2005).

[2] O. Sorlin and M.-G. Porquet, Prog. Part. Nucl. Phys. 61, 602 (2008).
[3] S. Pain et al., Phys. Rev. Lett. 96, 032502 (2006).

[4] Z. Dombrádi et al., Phys. Rev. Lett. 96, 182501 (2006).

[5] C. Campbell et al., Phys. Rev. Lett. 97, 112501 (2006). 
[6] A. Jungclaus et al., Phys. Rev. Lett. 99, 132501 (2007).

[7] I. Dillmann et al., Phys. Rev. Lett. 91, 162503 (2003).

[8] P. Federman et al., Phys. Lett. B140, 269 (1984).

[9] J. Schiffer and W. True, Rev. Mod. Phys. 48, 191 (1976).

[10] S. Pittel et al., Phys. Rev. C 48, 1050 (1993).

[11] T. Otsuka et al., Phys. Rev. Lett. 97, 162501 (2006).

[12] A. De Maesschalck, Ph.D. thesis, University of Ghen (2006) (to be published).

[13] O. Perru et al., Phys. Rev. Lett. 96, 232501 (2006).

[14] J. Van de Walle et al., Phys. Rev. Lett. 99, 142501 (2007).

[15] V. Fedoseyev et al., Hyp. Int. 127, 409 (2000).

[16] National Institute of Standards and Technology, http://physics. nist.gov/PhysRefData/IonEnergy/tblNew.html.

[17] National Nuclear Data Center, Brookhaven, http://www.nndc. bnl.gov/nndc/nudat/.

[18] F. Ames et al., Nucl. Instrum. Methods A 538, 17 (2005).

[19] F. Wenander et al., Nucl. Phys. A701, 528 (2002).

[20] O. Kester et al., Nucl. Instrum. Methods B 204, 20 (2003).

[21] U. Köster et al., AIP Conf. Proc. 798, 315 (2005).

[22] U. Köster et al., Spectrochim. Acta, Part B 58, 1047 (2003).

[23] M. Bernas et al., Nucl. Phys. A725, 213 (2003).

[24] U. Köster et al., Eur. Phys. J. A 15, 255 (2002).

[25] E. Bouquerel et al., Eur. Phys. J. ST 150, 277 (2007).

[26] U. Köster et al., Nucl. Instrum. Methods B (2008) (in press).

[27] J. Eberth et al., Prog. Part. Nucl. Phys. 46, 389 (2001).

[28] A. Ostrowski et al., Nucl. Instrum. Methods A 480, 448 (2002).

[29] D. Cline et al., Nucl. Phys. A133, 445 (1969).

[30] J. Daugas et al., Phys. Lett. B476, 213 (2000).

[31] J. Van Roosbroeck et al., Phys. Rev. C 70, 054307 (2005).
[32] K. Alder and A. Winther, Electromagnetic excitation: Theory of coulomb excitation with heavy ions (1975).

[33] T. Czosnyka et al., Bull. Am. Phys. Soc. 28, 745 (1983).

[34] D. Cline et al., Annu. Rev. Nucl. Part. Sci. 36, 683 (1986).

[35] N. Stone, At. Data Nucl. Data Tables 90, 75 (2005).

[36] S. Raman, At. Data Nucl. Data Tables 78, 1 (2001).

[37] L. Svensson et al., Nucl. Phys. A584, 547 (1995).

[38] E. Padilla-Rodal et al., Phys. Rev. Lett. 94, 122501 (2005).

[39] B. Kotliski et al., Nucl. Phys. A519, 646 (1990).

[40] S. Leenhardt et al., Eur. Phys. J. A 14, 1 (2002).

[41] M. Koizumi et al., Nucl. Phys. A730, 46 (2004).

[42] O. Kenn et al., Phys. Rev. C 65, 034308 (2001).

[43] J. Leske et al., Phys. Rev. C 71, 034303 (2005).

[44] M. Koizumi et al., Eur. Phys. J. A 18, 87 (2003).

[45] K. Langanke et al., Nucl. Phys. A757, 360 (2005).

[46] F. Iachello and A. Arima, The Interacting Boson Model (1987).

[47] J. Wilets and M. Jean, Phys. Rev. C 102, 788 (1956).

[48] A. Lisetskiy et al., Phys. Rev. C 70, 044314 (2004).

[49] M. Hjorth-Jensen et al., Phys. Rep. 261, 125 (1995).

[50] F. Nowacki, Ph.D. thesis, IReS Strasbourg (1996).

[51] N. Smirnova et al., Phys. Rev. C 69, 044306 (2004).

[52] E. Caurier et al., Acta Phys. Pol. B 30, 705 (1999).

[53] M. Honma et al., Phys. Rev. C 69, 034335 (2004).

[54] T. Otsuka et al., Phys. Rev. Lett. 81, 1588 (1998).

[55] A. Brown and A. Lisetskiy (private communication, 2008).

[56] M. Hasegawa et al., Nucl. Phys. A789, 46 (2007).

[57] X. Ji and B. Wildenthal, Phys. Rev. C 40, 389 (1989).

[58] D. Verney et al., Phys. Rev. C 76, 054312 (2007).

[59] Y. Zhang et al., Phys. Rev. C 70, 024301 (2004).

[60] J. Hakala et al., Phys. Rev. Lett. 101, 052502 (2008). 